\title{
Tumor Necrosis Factor-Alpha Is Produced by Dying Retinal Neurons and Is Required for Müller Glia Proliferation during Zebrafish Retinal Regeneration
}

\author{
Craig M. Nelson, Kristin M. Ackerman, Patrick 0’Hayer, Travis J. Bailey, Ryne A. Gorsuch, and David R. Hyde \\ Department of Biological Sciences and the Center for Zebrafish Research, Galvin Life Science Building, University of Notre Dame, Notre Dame, Indiana \\ 46556
}

\begin{abstract}
Intense light exposure causes photoreceptor apoptosis in dark-adapted adult albino zebrafish (Danio rerio). Subsequently, Müller glia increase expression of the Achaete-scute complex-like 1a (Ascl1a) and Signal transducer and activator of transcription 3 (Stat3) transcription factors and re-enter the cell cycle to yield undifferentiated neuronal progenitors that continue to proliferate, migrate to the outer nuclear layer, and differentiate into photoreceptors. A proteomic analysis of light-damaged retinal homogenates, which induced Müller glia proliferation when injected into an undamaged eye, revealed increased expression of tumor necrosis factor $\alpha$ (TNF $\alpha$ ) signaling proteins relative to undamaged retinal homogenates. TNF $\alpha$ expression initially increased in apoptotic photoreceptors and later in Müller glia. Morpholino-mediated knockdown of TNF $\alpha$ expression before light damage diminished the expression of both Asclla and Stat3 in Müller glia and significantly reduced the number of proliferating Müller glia without affecting photoreceptor cell death. Knockdown of TNF $\alpha$ expression in the Müller glia resulted in fewer proliferating Müller glia, suggesting that Müller glial-derived TNF $\alpha$ recruited additional Müller glia to re-enter the cell cycle. While TNF $\alpha$ is required for increased Asclla and Stat3 expression, Asclla and Stat3 are both necessary for TNF $\alpha$ expression in Müller glia. Apoptotic inner retinal neurons, resulting from intravitreal injection of ouabain, also exhibited increased TNF $\alpha$ expression that was required for Müller glia proliferation. Thus, TNF $\alpha$ is the first molecule identified that is produced by dying retinal neurons and is necessary to induce Müller glia to proliferate in the zebrafish retinal regeneration response.
\end{abstract}

\section{Introduction}

The teleost retina is a model for studying cellular and molecular mechanisms underlying retinal regeneration (Brockerhoff and Fadool, 2011; Fleisch et al., 2011; Nelson and Hyde, 2012). Following neuronal death, Müller glia undergo cell division to yield neuronal progenitors that continue to proliferate, migrate, and differentiate into the lost retinal cell types (Yurco and Cameron, 2005; Fausett and Goldman, 2006; Bernardos et al., 2007; Fimbel et al., 2007; Kassen et al., 2007; Thummel et al., 2008a). While avian and mammalian Müller glia exhibit limited proliferation, they cannot regenerate significant numbers of neurons and restore vision (Rowan and Cepko, 2004; Bringmann et al., 2006; Fischer et al., 2009; Karl and Reh, 2010). Therefore, identifying the signaling events required for zebrafish retinal regenera-

Received Aug. 11, 2012; revised Feb. 8, 2013; accepted March 4, 2013.

Author contributions: C.M.N., K.M.A., T.J.B., R.A.G., and D.R.H. designed research;C.M.N., K.M.A.,P.O., T.J.B., and R.A.G. performed research; C.M.N., K.M.A., P.O., T.J.B., R.A.G., and D.R.H. analyzed data; C.M.N., K.M.A., T.J.B., R.A.G., and D.R.H. wrote the paper.

This study was supported by a grant from the National Eye Institute-National Institutes of Health to D.R.H. (R01-EY018417) and the Center for Zebrafish Research, University of Notre Dame. We appreciate the assistance of the Freimann Life Science technicians for their care of the zebrafish and members of the Hyde lab for thoughtful discussions.

The authors declare no competing financial interests.

Correspondence should be addressed to Dr. David R. Hyde, Department of Biological Sciences, 027 Galvin Life Science Building, University of Notre Dame, Notre Dame, IN 46556. E-mail: dhyde@nd.edu.

DOI:10.1523/JNEUROSCI.3838-12.2013

Copyright $\odot 2013$ the authors $\quad 0270-6474 / 13 / 336524-16 \$ 15.00 / 0$ tion could yield approaches to induce mammalian retinal regeneration.

Several proteins have been identified that are necessary to regenerate the damaged zebrafish retina (Fausett et al., 2008; Thummel et al., 2008a; 2010; Calinescu et al., 2009; Qin et al., 2009; Ramachandran et al., 2010; Nelson et al., 2012; Powell et al., 2012; Wan et al., 2012). Heparin-binding epidermal-like growth factor (HB-EGF) is necessary and sufficient to induce Müller glia proliferation (Wan et al., 2012). However, HB-EGF is not expressed in damaged neurons, suggesting that unidentified molecules or processes induce Müller glia proliferation in the damaged zebrafish retina.

Tumor necrosis factor $\alpha(\mathrm{TNF} \alpha)$ is a secreted proinflammatory cytokine that signals several cellular processes, including apoptosis, cell survival, and proliferation (Tartaglia et al., 1993; Cheng, 1994; Chan and Leonard, 2000; Gupta, 2001). TNF $\alpha$ signaling activates pro-mitotic Stat proteins (Guo et al., 1998; Wang et al., 2000; Do et al., 2010), including Stat3 in a variety of cells and tissues (Guo et al., 1998; MacEwan, 2002; Miscia et al., 2002; Romanatto et al., 2007; Mori et al., 2011). TNF $\alpha$ activation of Stat 3 promotes ex vivo human neuronal progenitor cell proliferation and gliogenesis (Peng et al., 2008, 2011). TNF $\alpha$ also stimulates Tumor necrosis factor associated protein-1 (TRAP1), which stabilizes the retinoblastoma protein during mitosis (Chen et al., 1996), regulates pro-mitotic gene expression (Liu et al., 2010), and mediates Stat3 phosphorylation and activation in the mouse brain (Kubota et al., 2009). 
To determine whether dying photoreceptors induced Müller glia proliferation, we analyzed light-damaged retinal homogenates that stimulated Müller glial cell division when injected into undamaged eyes. This homogenate contained several proteins with increased expression relative to undamaged retinal homogenates, including TRAP1. We therefore characterized TNF $\alpha$ expression in the light-damaged retina and demonstrated that TNF $\alpha$ is expressed in apoptotic photoreceptors, followed by the Müller glia. TNF $\alpha$ was found to be necessary for both Stat 3 and Asclla expression, and Müller glia proliferation. Similarly, apoptotic inner retinal neurons and persistent Müller glia in the ouabain-damaged retina exhibit increased $\mathrm{TNF} \alpha$ expression, which is required for maximal Müller glia proliferation. Thus, TNF $\alpha$ is a general signal from apoptotic neurons to initiate Müller glia proliferation through the Asclla and Stat 3 proteins in the damaged zebrafish retina.

\section{Materials and Methods}

Fish maintenance. Wild-type $\mathrm{AB}$, albino, albino Tg[gfap:EGFP] nt11 (Kassen et al., 2007), and $\operatorname{Tg}[$ rho:Eco.NfsB-EGFP $]$ nt19 (Montgomery et al., 2010) lines of zebrafish (Danio rerio) were maintained in the Center for Zebrafish Research at the University of Notre Dame Freimann Life Science Center. The adult zebrafish used in these studies were 6-18 months old and $4-5 \mathrm{~cm}$ in length. Fish were maintained under a light and dark cycle of $14 \mathrm{~h}$ light ( $250 \mathrm{lux})$ and $10 \mathrm{~h}$ of dark at $28.5^{\circ} \mathrm{C}$. All experimental protocols in this study were approved by the University of Notre Dame Animal Care and Use Committee and are in compliance with the Association for Research in Vision and Ophthalmology statement for the use of animals in vision research.

Retinal damage paradigms. Constant intense-light treatment (Vihtelic and Hyde, 2000; Vihtelic et al., 2006) was used to kill rod and cone photoreceptors in adult albino zebrafish. The albino fish were darkadapted for $14 \mathrm{~d}$ and then placed in clear polycarbonate tanks between four $54 \mathrm{~W}$ mercury fluorescent bulbs producing a light intensity of 20,000 lux. The constant light was maintained for up to $4 \mathrm{~d}$, after which they were returned to standard light conditions.

Inner retinal neurons (ganglion cells, amacrine cells, and bipolar cells) were killed by intravitreal injection of ouabain at a final vitreal concentration of $2 \mu \mathrm{M}$ (Fimbel et al., 2007). A double-edged sapphire microknife (World Precision Instruments) was used to make a small incision in the posterior cornea adjacent to the lens and the appropriate volume (0.2$0.5 \mu \mathrm{l}$ ) of a freshly prepared ouabain solution (MP Biomedicals) was injected using a blunt-end 33 gauge Hamilton syringe.

Preparation and intravitreal injection of retinal lysates. Total retinal lysates were generated by isolating 40-60 retinas either from darkadapted controls or after $16 \mathrm{~h}$ of constant intense light and suspending them in $1.5 \mathrm{ml}$ centrifuge tubes kept frozen on dry ice containing $0.4 \mu \mathrm{l}$ of $1 \times$ PBS, pH 7.4, containing a protease inhibitor (Roche Diagnostics) per retina and then briefly sonicated. The recipient adult zebrafish were anesthetized in 2-phenoxyethanol (diluted 1:1000 in system water). A double-edged sapphire microknife was used to make a small incision in the cornea and $0.5 \mu$ l of homogenized retinal lysate was injected using a 33 gauge Hamilton syringe into the intravitreal space of the eye. The fish were revived and returned to standard light conditions.

Intravitreal injection of recombinant HB-EGF. Recombinant HB-EGF (R\&D Systems) was diluted in sterile $1 \times \mathrm{PBS}, 0.1 \%$ bovine serum albumin (BSA; vehicle) to a concentration of either $200 \mathrm{ng} / \mu \mathrm{l} \mathrm{or} 100 \mathrm{ng} / \mu \mathrm{l}$ as described previously (Wan et al., 2012). A small incision in the posterior cornea adjacent to the lens was created using a double-edged sapphire blade (World Precision Instruments). A blunt-end 33 gauge Hamilton syringe was used to inject either the PBS/0.1\% BSA vehicle solution (control) or $2 \mu \mathrm{l}$ of $100 \mathrm{ng} / \mu \mathrm{l}$ or $1 \mu \mathrm{l}$ of $200 \mathrm{ng} / \mu \mathrm{l} \mathrm{HB}$-EGF solution into the vitreous of the left eye of each fish for 3 consecutive days. At 2 or $3 \mathrm{~d}$ after the third injection, the fish were killed and the eyes were enucleated and processed for immunohistochemistry.

Proteomic analysis. Total dorsal retinal proteins were isolated from either 100 undamaged dorsal retinas or from $10016 \mathrm{~h}$ light-damaged dorsal retinas, suspended in $1.5 \mathrm{ml}$ centrifuge tubes, and kept frozen on dry ice containing $1 \mu \mathrm{l}$ of $1 \times$ PBS containing a protease inhibitor (Roche Diagnostics) and 1\% Triton X-100 per retina and then briefly sonicated. The homogenates were separately labeled with either $\mathrm{Cy} 3$ or $\mathrm{Cy} 5$, combined, and then separated by 2D gel electrophoresis (Applied Biomics). Changes in protein expression levels were quantified by comparing the fluorescence intensities of control and $16 \mathrm{~h}$ light damaged spots. Spots that increased in intensity by at least twofold were picked from the gels, trypsin digested, and analyzed by MALDI-TOF mass spectrometry (Applied Biomics). Combined use of peptide mass fingerprinting of the mass spectrometry spectra and peptide fragmentation mapping-derived sequences were analyzed by GPS Explorer software equipped with the MASCOT search engine to identify proteins from primary sequence databases. As noted in the text, the full list of identified proteins is found in Table 1.

Western blotting. Dorsal retinas were isolated, homogenized in $1 \times$ PBS/1\% Triton X-100 and protease inhibitor (Roche Diagnostics), and incubated on ice for $1 \mathrm{~h}$. Lysates were briefly centrifuged to remove Triton X-100-insoluble debris and the equivalent of two dorsal retinas was combined with $2 \times$ Laemmli sample buffer and $10 \times$ reducing agent (Invitrogen). Proteins were processed, electrophoresed, and transferred to Hybond PDVF membranes (GE Healthcare) as described previously (Nelson et al., 2012). The membranes were blocked in PBS/5\% nonfat dry milk $/ 0.1 \%$ Tween 20 for $1 \mathrm{~h}$ at room temperature and then incubated with either rabbit anti-TNF $\alpha$ (1:2000; AnaSpec) or mouse anti-actin (1:10,000; EMD Millipore) overnight at $4^{\circ} \mathrm{C}$. The membranes were washed, incubated with horseradish peroxidase-conjugated secondary antibodies (1:10,000; GE Healthcare), and detected using the ECL-Prime detection system (GE Healthcare) as described previously (Nelson et al., 2012).

Immunohistochemistry. Zebrafish were killed at designated times during treatment by an overdose of 2-phenoxyethanol anesthesia. Eyes were enucleated, fixed in either 9:1 ethanolic formaldehyde (100\% ethanol: $37 \%$ formaldehyde) or $4 \%$ PFA ( $4 \%$ paraformaldehyde, $5 \%$ sucrose, $1 \times$ PBS), processed, and sectioned as described previously (Thummel et al., 2010; Nelson et al., 2012).

Slides were incubated at $50^{\circ} \mathrm{C}$ for $20-30 \mathrm{~min}$ and then rehydrated in $1 \times$ PBS at room temperature for $20 \mathrm{~min}$. Sections that underwent antigen retrieval (labeling with either anti-PCNA or anti-Asclla on 4\% PFA fixed tissues) were boiled in $10 \mathrm{~mm}$ sodium citrate, $\mathrm{pH}$ 6.0, with $0.1 \%$ Tween 20 for $15 \mathrm{~min}$ and then cooled at room temperature (over $\sim 40$ $\mathrm{min}$ ). All retinal sections were blocked and incubated with primary antibodies overnight at $25^{\circ} \mathrm{C}$ as previously described (Thummel et al., 2010; Nelson et al., 2012). Primary antibodies used in this study were the mouse anti-PCNA monoclonal antibody (1:1000, clone PC10; Sigma Chemical), rabbit anti-PCNA polyclonal antiserum (1:750; Abcam), mouse anti-EGFP monoclonal antibody (1:1000; Invitrogen,), rabbit anti-EGFP polyclonal antiserum (1:1000; Invitrogen), rabbit anti-Stat3 polyclonal antiserum (1:100; Kassen et al., 2007), rabbit anti-Ascll polyclonal antiserum (1:50; Sigma-Aldrich), rabbit anti-TNF $\alpha$ polyclonal antiserum (1:30; AnaSpec), mouse monoclonal antibody 4C4 (1:250; generous gift from Dr. Jonathan Scholes, University College London), mouse anti-HuC/D monoclonal antibody (1:30; Invitrogen), rabbit antirhodopsin polyclonal antiserum (1:5000; Vihtelic et al., 1999), rabbit anti-UV opsin polyclonal antiserum (1:1000; Vihtelic et al., 1999), and rabbit anti-blue opsin polyclonal antiserum (1:250; Vihtelic et al., 1999). Sections were washed and incubated in secondary antibody diluted 1:500 in $1 \times$ PBS $/ 0.05 \%$ Tween 20 for $1 \mathrm{~h}$ at room temperature. Secondary antibodies used in this study were Alexa Fluor goat anti-primary IgG 488 , 568, 594, and 647 (1:500; Invitrogen). Nuclei were labeled with TOPRO-3 (Invitrogen) diluted 1:1000 in $1 \times$ PBS/0.05\% Tween 20. Slides were washed and mounted with glass coverslips and Vectashield mounting medium (Vector Laboratories).

Terminal deoxynucleotidyl transferase-mediated biotinylated UTP nick end labeling (TUNEL) was performed to identify apoptotic cells in frozen retinal sections using the ApoAlert DNA Fragmentation Assay kit (Clontech) in label buffer containing 2\% biotinylated dUTP (New England BioLabs) as previously described (Bailey et al., 2010). Sections were processed and dUTP was detected as described previously (Bailey et al., 2010). 
Table 1. Top ranked protein names and accession numbers

\begin{tabular}{|c|c|c|}
\hline $\begin{array}{l}\text { Spot } \\
\text { number }\end{array}$ & Top ranked protein name (Danio rerio) & $\begin{array}{l}\text { Accession } \\
\text { number }\end{array}$ \\
\hline 1 & PREDICTED: similar to AT motif binding factor 1 & gi|189521821 \\
\hline 2 & Heat shock protein 4, like & gi 47550833 \\
\hline 3 & Novel protein & gi 126632590 \\
\hline 4 & Heat shock protein $90 \mathrm{kDa}$ beta, member 1 & gi|38016165 \\
\hline 5 & PREDICTED: similar to LOC567732 protein & gi| 189528839 \\
\hline 6 & PREDICTED: similar to tripartite motif-containing 39 & gi|125821174 \\
\hline 7 & Complement component 9 & gi|220941693 \\
\hline 8 & Heat shock protein 5 & gi|39645428 \\
\hline 9 & Novel protein similar to heat shock cognate $70 \mathrm{kDa}$ & gi|169158081 \\
\hline 10 & Dynein, axonemal, heavy polypeptide 9 & gi 41055024 \\
\hline 11 & Transferrin-a & gi|220672954 \\
\hline 12 & Transferrin-a & gi|220672954 \\
\hline 13 & Sb:cb26 protein & gi|33417203 \\
\hline 14 & PREDICTED: similar to transketolase & gi 125829873 \\
\hline 15 & Hypothetical protein LOC767787 & gi|115529393 \\
\hline 16 & Hpx protein & gi| 112180551 \\
\hline 17 & Hpx protein & gi|112180551 \\
\hline 18 & Hpx protein & gi $\mid 112180551$ \\
\hline 19 & Hemopexin & gi| 162287365 \\
\hline 20 & $\begin{array}{l}\text { Serpin peptidase inhibitor, clade A (alpha-1 antiproteinase, } \\
\text { antitrypsin), member } 7\end{array}$ & gi|54400446 \\
\hline 21 & Pkm2a protein & gi|45501385 \\
\hline 22 & Hpx protein & gi $\mid 112180551$ \\
\hline 23 & Serpina1 protein & gi|38541769 \\
\hline 24 & $\begin{array}{l}\text { Novel protein similar to serine (or cysteine) proteinase inhibitor, } \\
\text { clade A (alpha-1 antiproteinase) }\end{array}$ & gi|55962788 \\
\hline 25 & Zgc:123103 & gi| 116284149 \\
\hline 26 & $26-29 \mathrm{kDa}$ proteinase protein & gi|49901179 \\
\hline 27 & Kinesin family member 14 & gi|113678899 \\
\hline 28 & S-antigen; retina and pineal gland (arrestin) & gi 76253800 \\
\hline 29 & Glyceraldehyde-3-phosphate dehydrogenase, spermatogenic & gi|47085833 \\
\hline 30 & $\begin{array}{l}\text { Novel protein similar to vertebrate phosphoglycerate mutase } \\
\text { family member } 4\end{array}$ & gi|220678446 \\
\hline 31 & Hypothetical protein LOC560055 & gi $\mid 154426250$ \\
\hline 32 & Novel pentaxin family domain containing protein & gi| 126540682 \\
\hline 33 & Apolipoprotein A-I & gi $\mid 18858281$ \\
\hline 34 & PREDICTED: hypothetical protein & gi| 189529246 \\
\hline 35 & Recoverin & gi|41152377 \\
\hline 36 & Hypothetical protein LOC322327 & gi|194578933 \\
\hline 37 & Hypoxia upregulated 1 & gi $\mid 47086637$ \\
\hline 38 & Heat shock protein HSP 90 beta & gi| 109835356 \\
\hline 39 & Platelet-derived growth factor receptor alpha & gi| 148726416 \\
\hline 40 & Novel protein similar to gelsolin, like 1 & gi|148725532 \\
\hline 41 & Aconitase 2, mitochondrial & gi|38707983 \\
\hline 42 & Keratin $18[$ D. rerio $]$ & gi|29335504 \\
\hline 43 & TNF receptor-associated protein 1 & gi|213627589 \\
\hline 44 & Radixin isoform 1 & gi|51972166 \\
\hline 45 & Guanine monphosphate synthetase & gi|220672808 \\
\hline 46 & Tyrosyl-tRNA synthetase, cytoplasmic & gi 109895056 \\
\hline 47 & Hypothetical protein LOC767787 & gi|115529393 \\
\hline 48 & RuvB-like 2 & gi|27819634 \\
\hline 49 & Zgc:123103 & gi 157422889 \\
\hline 50 & Brain creatine kinase $B$ & gi|27545193 \\
\hline 51 & PREDICTED: similar to transketolase & gi| 125829873 \\
\hline 52 & Ceruloplasmin & gi|94732872 \\
\hline
\end{tabular}

In situ hybridization. Total RNA was isolated from zebrafish embryos at $48 \mathrm{~h}$ postfertilization (hpf) using Trizol (Invitrogen) and then reverse transcribed using random primers with the Superscript III Preamplification System (Invitrogen). The tnf $\alpha$ cDNA was amplified using Platinum Taq (Invitrogen) and two primers (Forward 5' GCTGGTGATGGTGTCTAGGAGGA 3' and Reverse 5'TGTTGGCGGCACATTGCCAAGA $\left.3^{\prime}\right)$ with an annealing temperature of $58^{\circ} \mathrm{C}$. The $\sim 750$ bp PCR product was gel purified (QIAquick Gel Extraction; Qiagen), cloned into pCRIITOPO, and then sequenced to confirm the identity of the $t n f \alpha$ cDNA.
The tnf $\alpha$ cDNA was subcloned into the pCRII-TOPO (Invitrogen) vector and antisense and sense digoxigenin (DIG)-labeled RNA probes were in vitro transcribed (Roche DIG RNA Labeling Kit SP6/T7). The tnf $\alpha$ cDNA plasmid was linearized with either BamHI (antisense probe) or NotI (sense probe), precipitated, and in vitro transcribed with either T7 or SP6 RNA polymerase, respectively. The in vitro transcription reactions were terminated by adding $0.2 \mathrm{M}$ EDTA and the riboprobes were precipitated using ammonium acetate and $100 \%$ ethanol. The quality of the in vitro transcribed RNA was confirmed by electrophoresis through a $1 \%$ agarose formaldehyde gel.

The $h b$-egfa cDNA was synthesized from total RNA isolated from undamaged retinas using Trizol and reverse transcribed as described above. The single-stranded DNA was PCR amplified using Platinum TaqPCR, the $h b$-egfa Forward primer TGAGTTCTCTGGGGATTATGGG and the Reverse primer ATTAGGGCAGGACGAAGTTGGT (Wan et al., 2012) at an annealing temperature of $55.5^{\circ}$. The $982 \mathrm{bp} h b$-egfa cDNA was gel purified and cloned into the pCRII-TOPO vector. The antisense and sense DIG-labeled RNA probes were synthesized in vitro using BamHIlinearized vector and T7 RNA polymerase (antisense probe) or NotIlinearized vector and SP6 RNA polymerase (sense probe).

Whole eyes were fixed overnight at $4^{\circ} \mathrm{C}$ in $4 \%$ PFA, cryopreserved, and sectioned at $14 \mu \mathrm{m}$. In situ hybridization was performed on retinal cryosections as previously described (Hitchcock and Kakuk-Atkins, 2004) with minor modifications. The procedure was performed at room temperature unless otherwise noted. Retinal sections were thawed from $-80^{\circ} \mathrm{C}$ to room temperature for $1 \mathrm{~h}$ and rehydrated in an ethanol/DEPC water series of $100,95,70$, and $50 \%$ for $1 \mathrm{~min}$ each. The slides were washed in $2 \times$ SSC for $1 \mathrm{~min}$ followed by a proteinase $\mathrm{K}$ ( $30 \mu \mathrm{l}$ of 20 $\mathrm{mg} / \mathrm{ml}$ stock into $50 \mathrm{ml}$ of buffer $[0.1 \mathrm{M}$ Tris- $\mathrm{HCl}, \mathrm{pH} 8.0$, and $50 \mathrm{~mm}$ EDTA]) treatment at $37^{\circ} \mathrm{C}$ for $4.5 \mathrm{~min}$. The proteinase $\mathrm{K}$ was washed off with DEPC water, the sections treated with $0.1 \mathrm{~m}$ triethanolamine (TEA) for $3 \mathrm{~min}$, and then incubated in $0.1 \mathrm{M}$ TEA with acetic anhydride for 10 min. The slides were dehydrated in an ethanol/DEPC series: $2 \times$ SSC, $50 \%$ ethanol, $70 \%$ ethanol, $95 \%$ ethanol, and twice with 100\% ethanol. Sections were dried out at room temperature for $3 \mathrm{~h}$. The probe was diluted into $2 \mathrm{ml}$ of hybridization buffer $(200 \mu \mathrm{l}$ of $10 \times$ salt $[\mathrm{NaCl}$, Tris- $\mathrm{HCl}$, Tris-Base, sodium phosphate monobasic anhydrous, sodium phosphate dibasic monohydrous], $1 \mathrm{ml}$ of deionized formamide, $400 \mu \mathrm{l}$ $50 \%$ dextran sulfate, $200 \mu \mathrm{l}$ tRNA $(50 \mathrm{mg} / \mathrm{ml}), 40 \mu \mathrm{l} 50 \times$ Denhardt's solution, and $160 \mu \mathrm{l}$ DEPC water) and heated to $95^{\circ} \mathrm{C}$ for $5 \mathrm{~min}$, snap cooled on ice for $2 \mathrm{~min}$, placed over sections (100 $\mu \mathrm{l}$ at $1 \mathrm{ng} / 1 \mu \mathrm{l})$, and coverslipped with HybridSlips (Grace Bio-Labs). Sections were hybridized with riboprobe overnight at $55^{\circ} \mathrm{C}$ in a humidity chamber. After hybridization, the sections were washed three times with a 1:1 ratio of $2 \times$ SSC and deionized formamide plus $0.1 \%$ Tween 20 at $55^{\circ} \mathrm{C}$ for $30 \mathrm{~min}$ each, rinsed with MABT (100 mm maleic acid, $\mathrm{pH} 7.5,150 \mathrm{~mm} \mathrm{NaCl}$, $0.1 \%$ Tween 20) for $30 \mathrm{~min}$ at $55^{\circ} \mathrm{C}$, rinsed in MABT for $30 \mathrm{~min}$ at room temperature, and then blocked in MABT with $10 \%$ heat-inactivated sheep serum for $1 \mathrm{~h}$. Sections were incubated overnight in a dark humidity chamber at $4^{\circ} \mathrm{C}$ with anti-DIG AP fragments (Roche)/MABT diluted 1:1500. After antibody incubation, the sections were washed three times for $20 \mathrm{~min}$ with MABT, washed twice for $10 \mathrm{~min}$ with stain buffer (100 mM Tris- $\mathrm{HCl}, 100 \mathrm{~mm} \mathrm{NaCl}, 50 \mathrm{~mm} \mathrm{MgCl}_{2}, \mathrm{pH}>9.0$ ), and then processed with $22.5 \mu \mathrm{l} \mathrm{NBT} / 17.5 \mu \mathrm{l}$ BCIP (both $50 \mathrm{mg} / \mathrm{ml}$ stock solution) into $5 \mathrm{ml}$ of stain buffer at room temperature. The reaction was stopped with $0.1 \mathrm{M}$ Tris-HCl, coverslipped, and imaged on a Leica DM 5500B.

Confocal microscopy and statistical analysis. Confocal microscopy was performed with a Leica TCS SP5 confocal microscope. Only retinal sections containing or immediately adjacent to the optic nerve were examined to maintain consistency across experimental and control groups. Labeled cell counts from retinal sections were obtained from either 5- $\mu$ m-thick confocal $z$-stacks (Stat3 and Asclla immunolabeling) or 5to 8 - $\mu \mathrm{m}$-thick confocal $z$-stacks (all other immunolabeling) that began at the surface of the $14 \mu \mathrm{m}$ tissue sections closest to the coverslip using either the entire retina (retinal lysate injection experiments) or a $350 \mu \mathrm{m}$ linear distance across the dorsal retina centered between the circumferential marginal zone and the optic nerve that consistently received the same degree of damage and reliably resulted in comparable subsequent high levels of Müller glia proliferation (Thomas et al., 2012). Between 10 
and 15 experimental and control retinas were examined for each experimental time point. Low-intensity signals were enhanced and background signals were reduced in representative images using the levels function in Adobe Photoshop (Adobe Systems). Levels were adjusted identically to all layers within a panel and to all panels in a figure.

Statistical significance was calculated by using a Student's $t$ test (twotailed and two-sample unequal variance). The data met the assumptions of the test in all instances. Actual $p$ values are found in the text, with $p$ values $<0.05$ and 0.01 being considered significant and highly significant, respectively.

Electroporation of morpholinos into the adult zebrafish retina. Lissaminetagged morpholinos (Gene Tools) used in this study included: anti-tnf $\alpha$ morpholino (5'-AGCTTCATAATTGCTGTATGTCTTA-3') that is complementary to the $5^{\prime}$ UTR of $\operatorname{tnf} \alpha$ (Matthews et al., 2009), anti-tnf $\alpha$ 5-base mismatch control morpholino (5'-ACCTTGATAATTCCTCTATCTCT TA- $3^{\prime}$ ) that has the mismatched bases italicized, anti-stat3 morpholino that is complementary to the $5^{\prime}$ UTR of stat3 (Kassen et al., 2009), anti-asclla morpholino that is complementary to the 5' UTR of asclla (Fausett et al., 2008), anti-lin28a that is complementary to the 5' UTR of lin28a (Ramachandran et al., 2010), anti-hb-egfa morpholino that is complimentary to the 5' UTR of $h b$-egfa (Wan et al., 2012), or a Standard Control morpholino (Gene Tools) that is not complementary to any known sequence in the zebrafish genome. Morpholinos were resuspended in water at a working concentration of $3 \mathrm{~mm}$ and electroporated into the adult zebrafish retina as previously described (Thummel et al., 2008a). The electroporated fish were revived before being placed into constant intense light or injected with ouabain.

Quantitative real-time PCR. Total RNA was isolated from dorsal retinas collected before and at $16 \mathrm{~h}$ of constant light damage using the RNAqueous (Ambion) protocol and reagents. The cDNA was reverse transcribed by Superscript III first strand synthesis (Invitrogen) and quantified using a NANODROP spectrophotometer (Thermo Scientific). The gene-specific primers used for stat 3 and asclla amplification were described previously (Nelson et al., 2012). The protocols for sample preparation and run conditions described in the ABI 7500 (Applied Biosystems) user manual were followed and described previously (Nelson et al., 2012). Agarose gel electrophoresis and dissociation curve analysis verified that single products were produced with each of the primer pairs used in these experiments. The $\Delta \Delta$ CT method was used for data analysis as described previously (Kassen et al., 2007). The $18 \mathrm{~S}$ rRNA was used as the reference gene $(\Delta \mathrm{CT})$ and three biological replicates were performed in three technical replicates. The normalized $\Delta \mathrm{CT}$ values for each gene from each time point were normalized against the control group to yield the $\Delta \Delta \mathrm{CT}$, which were used to calculate the $\log _{2}$-fold change in gene expression levels.

\section{Results}

\section{Photoreceptor cell death produces a factor that induces Müller glia proliferation}

Previous studies demonstrated that very low numbers of Müller glia actively divide in the undamaged adult zebrafish retina (Thummel et al., 2008a). Following light-induced photoreceptor cell death, there is a significant increase in the number of Müller glia that re-enter the cell cycle (Vihtelic and Hyde, 2000; Thummel et al., 2008a). One potential mechanism for this induction of Müller glia proliferation is that dying photoreceptors express a factor that induces Müller glia to re-enter the cell cycle. We hypothesized that this Müller glia proliferation inducing factor would be present at increased levels when photoreceptor apoptosis is maximal.

To determine the time of maximal photoreceptor cell death, we examined TUNEL during the first $31 \mathrm{~h}$ of light damage. While the dark-adapted control retinal sections contained $4.8 \pm 2.5$ TUNEL-positive nuclei in the outer nuclear layer (ONL), the retinas that had been light treated for 16 and $24 \mathrm{~h}$ possessed $88 \pm$ 9 and $82 \pm 13$ TUNEL-positive nuclei in the ONL, respectively (data not shown). By $31 \mathrm{~h}$ of constant light, the number of TUNEL-positive nuclei decreased to $48 \pm 16$. Consistent with previous results (Vihtelic et al., 2006), maximal photoreceptor apoptosis was observed at 16 and $24 \mathrm{~h}$ of constant light exposure, which represents the time that the potential photoreceptorderived factor that induces Müller glia proliferation was likely to be present. We selected the $16 \mathrm{~h}$ time point for analysis (over $24 \mathrm{~h}$ ) because the regeneration process had already started by $16 \mathrm{~h}$, with increased numbers of proliferating rod precursor cells and Stat3expressing Müller glia relative to the undamaged retina (Vihtelic and Hyde, 2000; Kassen et al., 2007).

To determine whether the light-damaged retina expressed a factor that induced Müller glia proliferation, retinas that were either undamaged (control) or light damaged for $16 \mathrm{~h}$ were homogenized and separately injected into the vitreous of undamaged eyes (Fig. 1). Retinal sections containing the optic nerve from 10 individual eyes were labeled for rhodopsin expression to assess the morphology of rod photoreceptors at 1,2 , and $3 \mathrm{~d}$ post homogenate injection (dpi; Fig. 1A-F, red). The rhodopsin expression pattern did not change significantly from 1-3 dpi in either control or light-damaged retinal lysates indicating that neither the injection nor the lysates damaged the rods. The strong rhodopsin labeling in the intravitreal space at 1 and 2 dpi confirmed that the donor homogenate was successfully injected and persisted in the recipient eyes (Fig. $1 A, B, D, E$ ). PCNA-positive nuclei within the rhodopsin-positive homogenate in the vitreous colocalized with the 4C4 antibody, confirming that these are microglial cells (data not shown).

To confirm that neither the light-damaged homogenate nor the injections resulted in retinal cell death, which would lead to Müller glia proliferation, we examined TUNEL at the three time points. Low numbers of TUNEL-positive cells were found at all three time points in the light-damaged retinal homogenateinjected eyes (Fig. 1J-L, green). We quantified the number of TUNEL-positive nuclei in the uninjected control retina, the undamaged retinal homogenate-injected eyes, and the lightdamaged retinal homogenate-injected eyes. There were no statistically significant differences in the number of TUNELpositive nuclei in any of these retinas at the different time points (Fig. 1O). These data confirmed that neither the homogenate nor the injection resulted in any significant retinal cell death.

The retinal sections were colabeled for PCNA expression to identify PCNA-positive cells in both the ONL and inner nuclear layer (INL; Fig. 1A-F, green, arrowheads, and arrows, respectively). The number of proliferating ONL rod precursor cells was not statistically different between the uninjected control eyes and the undamaged retinal homogenate-injected eyes (Fig. 1M, red and blue bars, respectively). Similarly, there was no statistical difference in the number of PCNA-positive ONL cells in the undamaged retinal homogenate-injected eyes and the light-damaged retinal homogenate-injected eyes at any of the three time points (Fig. 1M; blue and green bars, respectively). In contrast, the light-damaged retinal homogenate-injected eyes had significantly more PCNApositive INL cells than either the uninjected control or the undamaged retinal homogenate-injected eyes at 2 and $3 \mathrm{dpi}$ (Fig. $1 \mathrm{~N}$; green, red, and blue bars, respectively). At 2 and 3 dpi, the light-damaged retinal homogenate-injected retinas possessed $8.2 \pm 4.6$ and $16.6 \pm$ 4.1 PCNA-positive INL nuclei relative to $2.8 \pm 1.8$ and $4.1 \pm 3.4$ PCNA-positive INL nuclei in the undamaged retinal homogenateinjected eyes at 2 and $3 \mathrm{dpi}$, respectively $(p<0.005,0.0001$, respectively). The location and morphology of the PCNA-positive nuclei in the damaged retinal homogenate-injected retinas are consistent with that of Müller glia. Furthermore, the light-damaged retinal homogenate-injected retinas possessed small clusters of PCNApositive nuclei at $3 \mathrm{dpi}$ that were similar in morphology to the clus- 

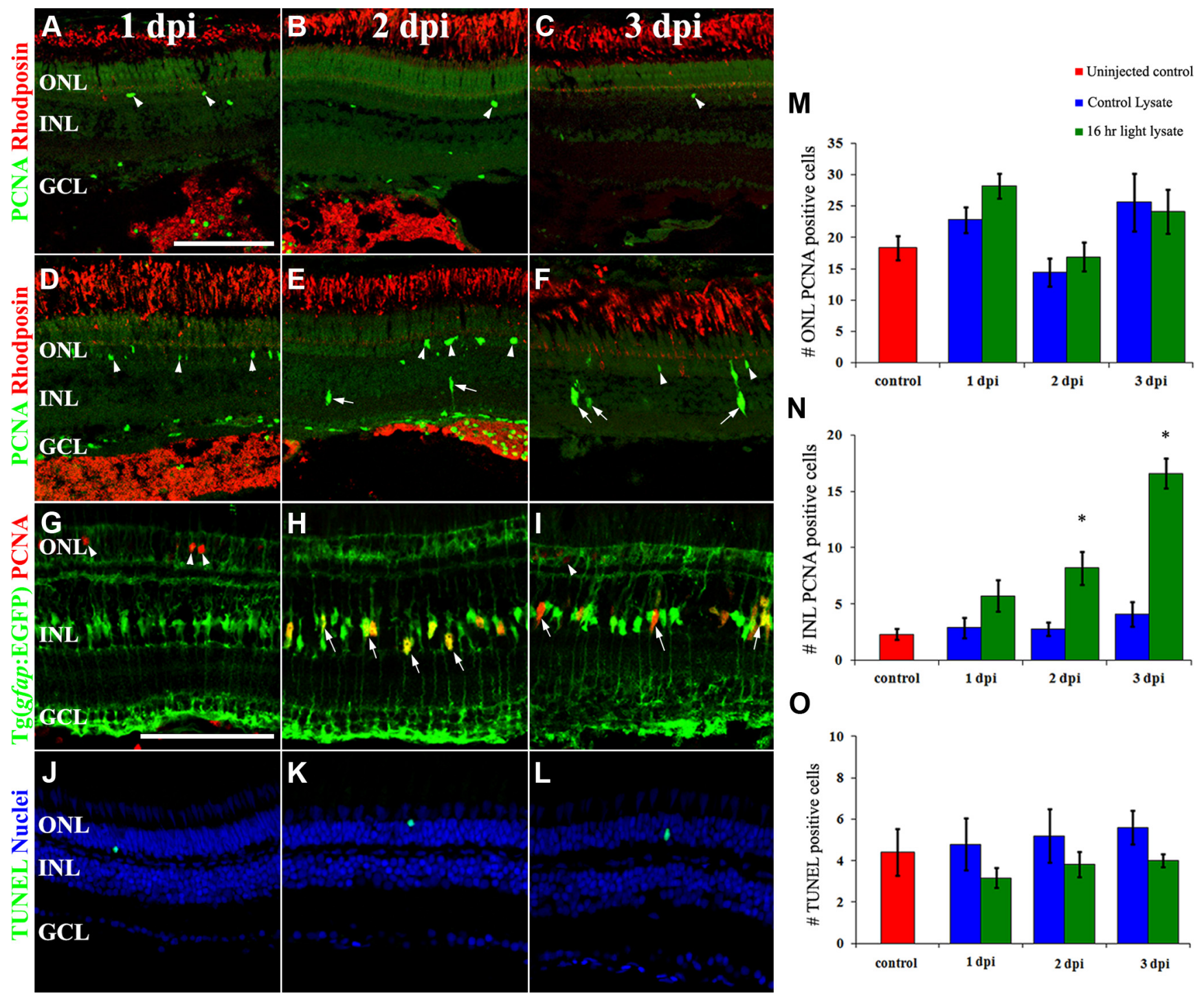

Figure 1. Light-damaged retinal homogenate contains a factor that induces Müller glia proliferation. Retinal sections from wild-type eyes that were intravitreally injected with a homogenate from either undamaged retinas $(\boldsymbol{A}-\boldsymbol{C}$ or light-damaged retinas $(\boldsymbol{D}-\boldsymbol{L})$ were immunolabeled with anti-rhodopsin (red) to visualize rod outer segments and anti-PCNA (green) to detect proliferating cells in the ONL and INL (arrowheads and arrows, respectively) at 1,2, and 3 dpi (days postinjection, $\boldsymbol{A}-\boldsymbol{F}$ ). Tg(gfap:EGFP)nt11 eyes were injected with light-damaged retinal homogenate and immunolabeled with anti-EGFP and anti-PCNA ( $\mathbf{G}-\mathbf{I}$, green and red, respectively) at 1,2 , and 3 dpi. The number of PCNA-positive cells significantly increased $(p<0.05, n=10)$ in the INL of light-damaged homogenate-injected eyes at 2 and 3 dpi relative to the controls $(\boldsymbol{N})$, but not in the ONL $(\boldsymbol{M})$. Injection of the light-damaged retinal homogenate did not cause any cell death as measured by the number of TUNEL-positive cells $(\boldsymbol{J}-\boldsymbol{L}, \mathbf{0})$. Scale bars: (in $\boldsymbol{A}) \boldsymbol{A}-\boldsymbol{F}, \boldsymbol{J}-\boldsymbol{L}, 50 \mu \mathrm{m}$; (in $\mathbf{G}) \mathbf{G}-\boldsymbol{I}, 50 \mu \mathrm{m}$.

ters of proliferating INL neuronal progenitors in the light-damaged retina (Vihtelic and Hyde, 2000; Kassen et al., 2007).

To confirm that the PCNA-positive INL cells in the lightdamaged retinal homogenate-injected retinas were Müller glia, we injected the light-damaged homogenate into the Tg(gfap:EGFP)nt11 transgenic line (Fig. 1G-I, green), which expresses EGFP specifically in Müller glia (Kassen et al., 2007). At 2 dpi, when we first observed an increased number of PCNA-positive cells, the PCNA colocalized with the EGFPpositive Müller glia (Fig. $1 H$ ). Similarly at $3 \mathrm{dpi}$, the small clusters of PCNA-positive nuclei colocalized with diffuse EGFP-expression in the hypertrophied Müller glia (Fig. 1I). These observations demonstrated that the light-damaged retinal homogenate contained at least one factor that was sufficient to induce the Müller glia to re-enter the cell cycle in the absence of any significant retinal cell death.
TRAP1 is expressed at elevated levels in the light-damaged retinal homogenate

To identify proteins and candidate signaling pathways that were present in the light-damaged retinal homogenate that could induce Müller glia proliferation, we undertook a comparative proteomic analysis of the homogenates. Total dorsal retinal proteins were isolated from either undamaged retinas and labeled with $\mathrm{Cy} 3$ or from $16 \mathrm{~h}$ light-damaged retinas and labeled with Cy5. Both protein homogenates were combined and then separated by $2 \mathrm{D}$ gel electrophoresis. Greater than 50 protein spots, which were expressed at least twofold greater in the light-damaged retinal homogenate relative to the undamaged retinal homogenate, were isolated and analyzed via MALDI-TOF mass spectrometry to identify the proteins (Table 1). One of the proteins corresponded to TRAP1 (Table 1, Spot 43), which suggested that TNF $\alpha$ signaling was a candidate for inducing Müller glia proliferation. 


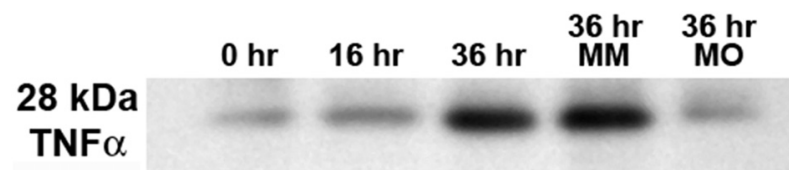

\section{$17 \mathrm{kDa}$ $\mathrm{TNF} \alpha$}

\section{Actin}

Figure 2. TNF $\alpha$ expression increases during constant light treatment. Total protein was isolated from dark-adapted albino retinas after 0 (Control), 16, and $36 \mathrm{~h}$ of constant light treatment. Immunoblotting for TNF $\alpha$ expression reveals a $28 \mathrm{kDa}$ band, likely corresponding to the uncleaved form of TNF $\alpha$. After $16 \mathrm{~h}$ of constant light, a $17 \mathrm{kDa}$ band is detected that corresponds to the mature, secreted form of TNF $\alpha$. Total protein was also isolated from darkadapted albino dorsal retinas that were injected and electroporated with either the tnf $\alpha$ 5-mis control morpholino (MM) or the tnf $\alpha$ morpholino (M0) and placed in constant intense light for $36 \mathrm{~h}$. While the tnf $\alpha$ 5-mis control retina had approximately the same amount of TNF $\alpha$ as the uninjected light-damaged retina ( $36 \mathrm{~h}$ ), the tnf $\alpha$ morphant possessed dramatically less TNF $\alpha$ protein. $\beta$-actin expression was detected as a loading control.

\section{TNF $\alpha$ expression is elevated in the light-damaged retina}

While TNF $\alpha$ was not one of the proteins analyzed by MALDITOF, we examined the expression of TNF $\alpha$ using an immunoblot with total protein homogenates from retinas that were undamaged and exposed to constant light for 16 and $36 \mathrm{~h}$. The control retinal lysates possessed a band of $28 \mathrm{kDa}$, which corresponded to the expected size of full-length pro-form zebrafish TNF $\alpha$ (Fig. 2). Following $16 \mathrm{~h}$ of constant light exposure, the expression of a 17 $\mathrm{kDa}$ protein, corresponding to the biologically relevant cleaved and secreted form of TNF $\alpha$, increased relative to the control (Fig. 2). After $36 \mathrm{~h}$ of constant light, both the 28 and $17 \mathrm{kDa}$ forms of TNF $\alpha$ were elevated (Fig. 2).

To determine the spatial expression of TNF $\alpha$ in the damaged retina, we immunolocalized the TNF $\alpha$ protein in the undamaged retina (Fig. 3A) and the light-damaged retina at $16 \mathrm{~h}$ of constant light (Fig. $3 B, D, E$ ) and at $36 \mathrm{~h}$ when the Müller glia begin proliferating (Fig. $3 C, F, G$ ). In control retinas, TNF $\alpha$ expression was observed within the outer plexiform layer (OPL) and the nerve fiber layer (NFL; Fig. 3A, arrowhead and double arrowhead, respectively). After $16 \mathrm{~h}$ of constant light, increased TNF $\alpha$ expression was associated with cells within the cone cell layer (Fig. $3 B$, arrows), ONL (open arrowhead), OPL (arrowhead), INL (double arrows), and NFL (double arrowhead). By 36 h of constant light, a similar TNF $\alpha$ expression pattern was observed, but the number and intensity of INL-labeled cells increased (Fig. 3C, double arrow) and the number and intensity of ONL and cone cell layer expression decreased (open arrowhead and arrow, respectively).

Because TNF $\alpha$ is a secreted protein and many different cells are associated with the OPL, we performed in situ hybridization to identify the cells that expressed the $\operatorname{tnf} \alpha$ transcript. In the undamaged retina, negligible amounts of $\operatorname{tnf} \alpha$ were detected in both the INL and ONL (Fig. 3). After $16 \mathrm{~h}$ of constant light, the expression of $t n f \alpha$ greatly increased in the ONL and, to a lesser extent, cells in the INL (Fig. 3I). After $36 \mathrm{~h}$ of constant light, the predominant expression of $t n f \alpha$ shifted from the ONL to the INL (Fig. $3 J)$. This suggests that the light damage initially results in in- creased $\operatorname{tnf} \alpha$ expression in the ONL photoreceptors, followed by increased expression within a subset of the INL cells.

To determine the identity of the cells that express TNF $\alpha$ during the constant light treatment, retinal sections were either immunolabeled with anti-TNF $\alpha$ antibodies (Fig. 3D-G) or in situ hybridized with an in vitro transcribed tnf $\alpha$ riboprobe (Fig. $3 K-$ $O)$. After $16 \mathrm{~h}$ of constant light, the TUNEL signal in the ONL occasionally colocalized with anti-TNF $\alpha$ immunolabeling (Fig. $3 D$, open arrowhead). Similarly, strong colocalization was observed between the $\operatorname{tnf} \alpha$ antisense probes (Fig. $3 K$, blue) and the TUNEL signal (green) within the ONL (arrows) and cone cell nuclei (arrowheads), which is consistent with the light-induced apoptotic rods and cones. Colocalization between TNF $\alpha$ antiserum (Fig. 3E, green) and the double-cone cell marker ZPR1 (red), as well as $t n f \alpha$ antisense probe (Fig. $3 L, M$, blue) with either Blue opsin immunolabeling (Fig. 3L, green) or EGFP expression (Fig. $3 M)$ in the rods of the Tg[rho:Eco. NfsB-EGFP $]$ nt19 transgenic line (Montgomery et al., 2010), confirmed that TNF $\alpha$ was expressed in both rod and cone photoreceptors at $16 \mathrm{~h}$ of constant light. No colocalization was observed between the microglial 4C4 immunolabeling (Fig. $3 G$, red; $O$, green) and either TNF $\alpha$ immunolabeling (Fig. 3G, green) or $\operatorname{tnf} \alpha$ antisense probes (Fig. 3O, blue). After $36 \mathrm{~h}$ of constant light, the $\operatorname{Tg}(g f a p$ :EGFP)nt11 transgenic line, which expresses EGFP in all Müller glia (Fig. $3 F, N$, green), exhibited TNF $\alpha$ protein (Fig. $3 F$, red) and $t n f \alpha$ mRNA (Fig. $3 N$, blue) expression in the INL Müller glia. Together, these results demonstrate that $\mathrm{TNF} \alpha$ expression increases initially in apoptotic rod and cone photoreceptors and then in Müller glia at a time when they are beginning to proliferate.

\section{TNF $\alpha$ expression in the light-damaged retina is required for} Müller glia proliferation, but not photoreceptor apoptosis To determine whether TNF $\alpha$ plays a role in the light-damaged retina, we intravitreally injected and electroporated lissaminetagged morpholinos into dark-adapted albino retinas immediately before placing the fish into constant intense light. One morpholino was designed to bind the $5^{\prime}$ untranslated region of $\operatorname{tnf} \alpha(\operatorname{tnf} \alpha \mathrm{MO})$ to block translation of the TNF $\alpha$ protein, while a control morpholino ( $\operatorname{tnf} \alpha 5$-mis) that contained five mismatched bases will not efficiently bind the $\operatorname{tnf} \alpha 5^{\prime}$ untranslated region.

After 16 and $36 \mathrm{~h}$ of constant intense light, the retinas were immunolabeled for TNF $\alpha$ expression (Fig. $4 A-D$, green). At $16 \mathrm{~h}$ of constant light, control retinas that were electroporated with the 5-base mismatch morpholino ( $t n f \alpha$ 5-mis) exhibited TNF $\alpha$ expression in photoreceptor outer segments (Fig. 4A, arrow), OPL (bracket), and the nerve fibers in the ganglion cell layer (GCL; asterisk). Following $36 \mathrm{~h}$ of constant light, the $\operatorname{tnf} \alpha$ 5-mis control morphant retinas revealed increased TNF $\alpha$ expression in the INL Müller glia (Fig. 4C, arrowheads). At both time points, light-damaged retinas that were not electroporated with a morpholino were indistinguishable from the tnf $\alpha$-mis control morphant retinas (data not shown). In contrast, the $t n f \alpha$ morphant retinas either lacked or possessed reduced TNF $\alpha$ expression in all of these retinal cell types or layers at 16 and $36 \mathrm{~h}$ of constant light (Fig. $4 B, D$, respectively). After $36 \mathrm{~h}$ of constant light, immunoblotting of protein lysates isolated from dorsal retinas showed similar expression of both the 28 and $17 \mathrm{kDa}$ TNF $\alpha$ protein bands in both controls, while the $\operatorname{tnf} \alpha$ morphant retinas exhibited a dramatic knockdown of both the 28 and $17 \mathrm{kDa}$ TNF $\alpha$ protein expression (Fig. 2).

To determine whether loss of TNF $\alpha$ signaling affected lightinduced photoreceptor cell death, uninjected, $\operatorname{tnf} \alpha$ 5-mis control and $t n f \alpha$ morphant retinas were labeled for TUNEL after $16 \mathrm{~h}$ of 


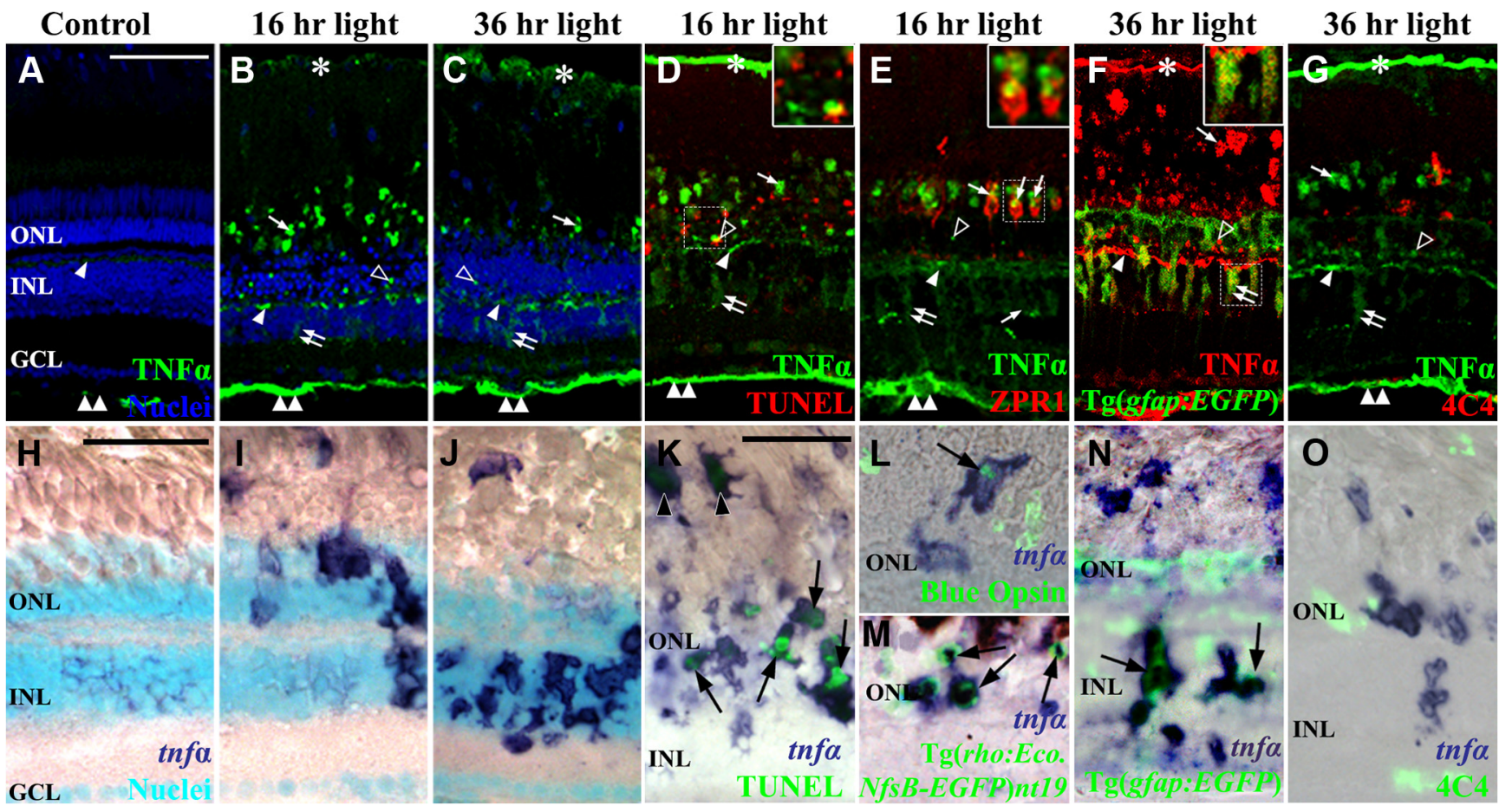

Figure 3. TNF $\alpha$ is expressed in different cell types during the constant light treatment. Dark-adapted albino zebrafish were exposed to constant intense light for $0 \mathrm{~h}$, $16 \mathrm{~h}$, or $36 \mathrm{~h}$. Cryosections were immunolabeled for TNF $\alpha(\boldsymbol{A}-\boldsymbol{E}, \mathbf{G}$, green; $\boldsymbol{F}$, red) or in situ hybridized with tnf $\alpha$ antisense riboprobe $(\boldsymbol{H}-\mathbf{0})$. TNF $\alpha$ antisera weakly labeled the NFL and 0 PL in undamaged control retinas $(\boldsymbol{A}$, double arrowhead and arrowhead, respectively). At 16 and $36 \mathrm{~h}$ of constant light ( $\boldsymbol{B}$ and $\boldsymbol{C}$, respectively), TNF $\alpha$ expression increased in the OPL (arrowhead), 0NL (open arrowhead), photoreceptor outer segments (arrow), INL (double arrow), NFL (double arrowhead), and retinal pigmented epithelium (RPE; asterisk). Following $36 \mathrm{~h}$ of constant intense light (C), TNF $\alpha$ antisera labeling in the INL reveals cells with processes that span from the GCL to the ONL (double arrow). At $16 \mathrm{~h}$ of light, TNF $\alpha$ colocalized with some TUNEL-positive 0NL cells ( $\boldsymbol{D}$, open arrowhead and inset) and ZPR1-positive double cones ( $\boldsymbol{E}$, arrows and inset). At $36 \mathrm{~h}$, TNF $\alpha$ colocalized with EGFP-positive Müller glia ( $\boldsymbol{F}$, double arrow and inset) in the Tg(gfap:EGFP)nt11 transgenic line, but not with 4C4-positive microglia $(\mathbf{G}$, red). In situ hybridization with a tnf $\alpha$ antisense probe $(\boldsymbol{H}-\mathbf{0}$, blue) demonstrates increased tnf $\alpha$ expression primarily in the 0 NL following $16 \mathrm{~h}$ of light $(\boldsymbol{I})$ and then primarily in the INL at $36 \mathrm{~h}$ of treatment $(\boldsymbol{J})$ relative to the undamaged control $(\boldsymbol{H})$. Colocalization of the tnf $\alpha$ antisense riboprobe with TUNEL-positive rods and cones $(\boldsymbol{K}$, arrows and arrowheads, respectively), blue opsin-expressing cones ( $\boldsymbol{L}$, arrow), and EGFP-expressing rod photoreceptors in the Tg[rho:Eco.NfsB-EGFP]nt19 transgenic line ( $\boldsymbol{M}$, arrows) occurred at $16 \mathrm{~h}$ of light damage. After $36 \mathrm{~h}$ of light damage, the EGFP-expressing Müller glia ( $\boldsymbol{N}$, green) in the Tg(gfap:EGFP)nt11 transgenic line expressed tnf $\alpha$ ( $\boldsymbol{N}$, arrows), while the 4C4-expressing microglia ( $\mathbf{0}$, green) did not express TNF $\alpha$. The boxed insets $(\boldsymbol{D}-\boldsymbol{F})$ are magnified 2.9-fold to highlight the colocalization with TNF $\alpha$. Scale bars: (in $\boldsymbol{A}) \boldsymbol{A}-\mathbf{G}, 50 \mu \mathrm{m}$; (in $\boldsymbol{H}) \boldsymbol{H}-\boldsymbol{J}, 50 \mu \mathrm{m}$; (in $\boldsymbol{K}) \boldsymbol{K}-\mathbf{0}, 50 \mu \mathrm{m}$.

constant light damage. Uninjected (data not shown) and thf $\alpha$ 5 -mis control retinal sections (Fig. 5A) contained $32.8 \pm 4.4$ and $28.9 \pm 3.7$ TUNEL-positive ONL nuclei respectively (Fig. 5C, blue and red bars, respectively). Similarly, the tnf $\alpha$ morphant retinas (Fig. $5 B$ ) possessed $30.5 \pm 4.5$ TUNEL-positive ONL nuclei (Fig. 5C). Thus, there was no significant difference in the number of TUNEL-positive cells in the $\operatorname{tnf} \alpha$ morphant retina relative to either of the control retinas ( $p=0.72$ and 0.79 , respectively), suggesting that TNF $\alpha$ is not required for light-induced photoreceptor cell death.

To examine if knockdown of TNF $\alpha$ affected cell proliferation in the light-damaged retina, dark-adapted albino zebrafish were either uninjected (data not shown) or injected and electroporated with either $\operatorname{tnf} \alpha$ 5-mis control morpholino or the $t n f \alpha$ morpholino and placed in constant intense light. The eyes were collected at specific time points and retinal sections were immunolabeled for PCNA-positive cells (Fig. 6A-H, green).

After $16 \mathrm{~h}$ of light damage, an average of $<2.0$ PCNA-positive cells were observed in the INL of the uninjected, $\operatorname{tnf} \alpha$ 5-mis control, and $t n f \alpha$ morphant retinal sections (Fig. 6I). Following $36 \mathrm{~h}$ of constant light damage, the $\operatorname{tnf} \alpha$ morphant retinal sections contained significantly fewer PCNA-positive INL cells $(4.3 \pm 1.2$; Fig. $6 F, I)$ compared with the uninjected $(26.7 \pm 3.7)$ and $t n f \alpha$ 5 -mis control sections $(21 \pm 3.5$; Fig. $6 I, p<0.001$ for each $)$. After 51 h of constant light, $t n f \alpha$ morphant retinas also contained significantly fewer PCNA-positive INL cells (9.1 \pm 2.7 ; Fig. 6G,I) relative to the uninjected $(38.2 \pm 4.5)$ and $\operatorname{tnf} \alpha$-mis control sections (28.4 \pm 4.4 ; Fig. $61, p<0.001$ for each). After $96 \mathrm{~h}$ of constant light, $t n f \alpha$ morphant retinas still contained significantly fewer PCNA-positive INL cells $(20.9 \pm 4.8$; Fig. $6 H, I)$ relative to the uninjected $(41.0 \pm 4.3)$ and $\operatorname{tnf} \alpha$ 5-mis control sections (40.6 \pm 4.4 ; Fig. $6 I, p<0.006$ for each). While there was no significant difference in the number of PCNA-positive ONL cells in the three treatments through 51 h of constant light, at $96 \mathrm{~h}$ of light the $\operatorname{tnf} \alpha$ morphant retinas contained significantly fewer PCNA-positive ONL cells $(11.5 \pm 2.8)$ relative to the uninjected $(67.1 \pm 5.3)$ and $t n f \alpha 5$-mis control sections (52.9 $\pm 7.4 ; p<0$. 001 for each). At $96 \mathrm{~h}$ of light, these PCNA-positive ONL cells likely correspond to both resident ONL rod precursor cells and INL-derived neuronal progenitors that migrated to the ONL. Thus, TNF $\alpha$ is required for the maximal proliferation of the INL Müller glia and neuronal progenitor cells beginning at $36 \mathrm{~h}$ of constant light.

The number of proliferating Müller glia is amplified through Müller glia expression of TNF $\alpha$

$\mathrm{TNF} \alpha$ is expressed at $16 \mathrm{~h}$ in the dying/damaged photoreceptors and then at $36 \mathrm{~h}$ in the Müller glia as they begin to proliferate (Fig. $3)$. Electroporation of the $\operatorname{tnf} \alpha$ morpholino at the start of constant light treatment inhibited TNF $\alpha$ expression in both the photoreceptors and Müller glia, resulting in a significant reduction in the number of PCNA-positive Müller glia (Fig. 6). To assess the 


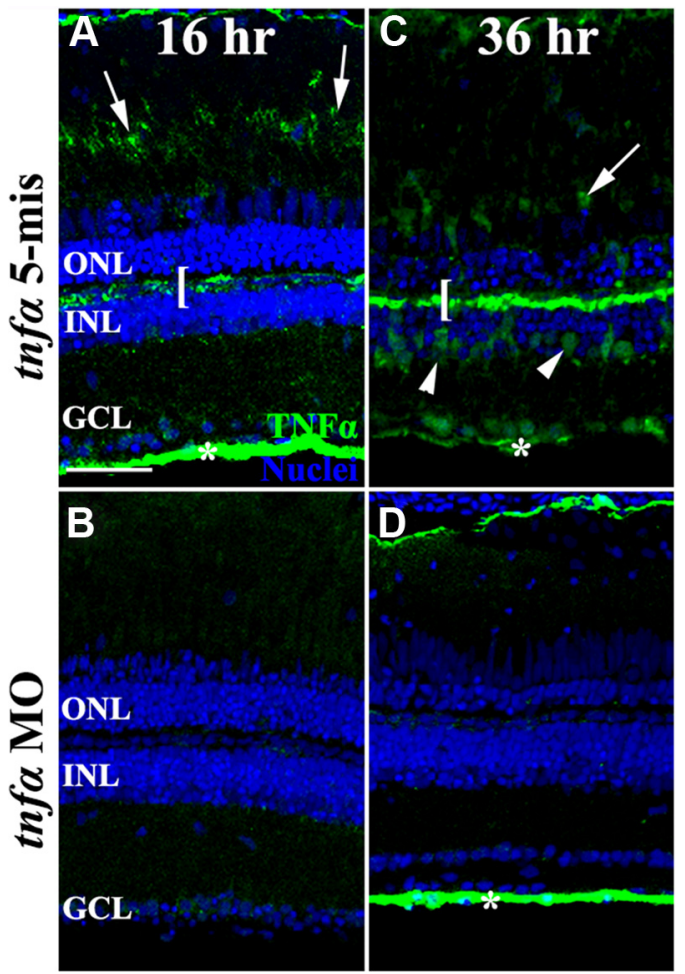

Figure 4. In vivo knockdown of TNF $\alpha$ during constant light treatment. Dark-adapted albino zebrafish were intravitreally injected and electroporated with either lissamine-tagged tnf $\alpha$ 5-mismatch morpholinos (tnf $\alpha$ 5-mis, A, C) or translation-inhibiting tnf $\alpha$ morpholinos (tnf $\alpha$ $\mathrm{MO}, \boldsymbol{B}, \boldsymbol{D})$ and then exposed to constant intense light for either $16 \mathrm{~h}(\boldsymbol{A}, \boldsymbol{B})$ or $36 \mathrm{~h}(\boldsymbol{C}, \boldsymbol{D})$. The tnf $\alpha$ morphant retinas showed decreased intensity of TNF $\alpha$ immunolabeling relative to both the uninjected (data not shown) and tnf $\alpha$-mis controls, in the outer segments (arrows), OPL (bracket), Müller glia (arrowheads), and the NFL (asterisk). Scale bar: (in A) A-D, $50 \mu \mathrm{m}$.
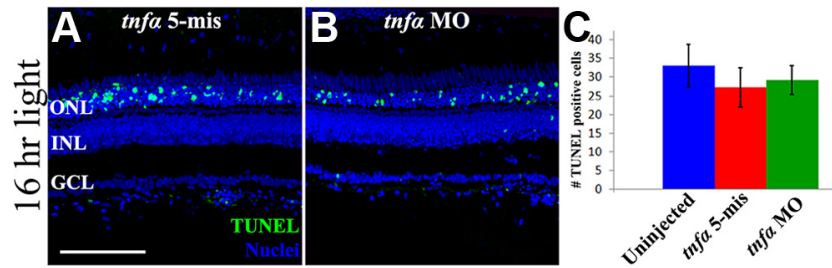

Figure 5. Knockdown of TNF $\alpha$ does not affect photoreceptor apoptosis during constant light treatment. Dark-adapted albino fish were intravitreally injected and electroporated with either lissamine-tagged tnf $\alpha$ 5-mismatch morpholinos (tnf $\alpha$ 5-mis, $A$ ) or translation inhibiting tnf $\alpha$ morpholinos (tnf $\alpha \mathrm{MO}, \boldsymbol{B})$ immediately before starting $16 \mathrm{~h}$ of constant light. Retinal cryosections were colabeled for TUNEL $(\boldsymbol{A}, \boldsymbol{B}$, green) and nuclei (To-Pro-3, blue) and the number of TUNEL-positive cells were quantified ( $C$ ). No significant difference was observed in the number of TUNEL-positive cells between the controls (blue and red bars) and the tnf $\alpha$ morphant (green bar, $p>0.05, n=10$ ). Scale bar: (in $\boldsymbol{A}) \boldsymbol{A}, \boldsymbol{B}, 50 \mu \mathrm{m}$.

role of TNF $\alpha$ expression primarily in the Müller glia, darkadapted albino zebrafish were either uninjected or injected and electroporated with either the $\operatorname{tnf} \alpha$ 5-mis control morpholino or the $\operatorname{tnf} \alpha$ morpholino into the retina following $16 \mathrm{~h}$ of light damage. The retinas were collected and labeled with anti-TNF $\alpha$ antisera after a total of $36 \mathrm{~h}$ of light treatment. The uninjected retinas (data not shown) were indistinguishable from the tnf $\alpha$-mis control retinas, with robust anti-TNF $\alpha$ labeling in both the OPL and the INL Müller glia (Fig. 7A, bracket and double arrows, respectively). In contrast, the $t n f \alpha$ morphant retinas had dramatically reduced TNF $\alpha$ labeling relative to the controls (Fig. 7E). Injection and electroporation of the $\operatorname{tnf} \alpha$ morpholino after $16 \mathrm{~h}$ of light treatment appears to have effectively knocked down
TNF $\alpha$ expression in the Müller glia, the apoptotic photoreceptors, and the NFL at $36 \mathrm{~h}$. Because the majority of TNF $\alpha$ expression in the apoptotic photoreceptors occurred at or before $16 \mathrm{~h}$ (Fig. 3B) and most of the TNF $\alpha$ expression in the Müller glia occurred after $16 \mathrm{~h}$ (Fig. $3 C$ ), the electroporation of the $\operatorname{tnf} \alpha$ morpholino after $16 \mathrm{~h}$ of light treatment primarily reduced TNF $\alpha$ expression in the Müller glia.

We knocked down the expression of TNF $\alpha$ after $16 \mathrm{~h}$ of intense light and examined PCNA expression in retinal sections after 36,51 , or $68 \mathrm{~h}$ of total constant light (Fig. $7 B-D, F-H$, green). After $36 \mathrm{~h}$ of light, the uninjected and $\operatorname{tnf} \alpha 5$-mis control morphant eyes contained significantly greater numbers of PCNA-positive INL cells $(28.8 \pm 2.4$ and $31.1 \pm 3.4$, respectively; Fig. 7I, blue and red bars, respectively), relative to the $\operatorname{tnf} \alpha$ morphant sections $(17.7 \pm 3.2$; Fig. $7 I$, green bars, $p=0.013$ and 0.009 , respectively). After $51 \mathrm{~h}$ of light damage, uninjected and tnf $\alpha$ 5-mis control retinas contained significantly more PCNApositive INL cells ( $38.5 \pm 4.0$ and $41.9 \pm 3.4$, respectively; Fig. $7 I$, blue and red bars, respectively) compared with the $\operatorname{tnf} \alpha$ morphant retinal sections (27.5 \pm 3.3 ; Fig. $7 I$, green bars, $p=0.047$ and 0.007 , respectively). By $68 \mathrm{~h}$ of light damage, uninjected and tnf $\alpha$ 5-mis control retinas contained a highly significant increase in the number of PCNA-positive INL cells (74.3 \pm 7.4 and $68.9 \pm$ 5.3, respectively; Fig. 7I, blue and red bars, respectively) relative to the $\operatorname{tnf} \alpha$ morphant retinal sections (36.5 \pm 6.1 ; Fig. $7 I, p<$ 0.001 for each).

To determine whether this significant reduction in the number of PCNA-positive cells was due to either fewer Müller glia re-entering the cell cycle or reduced numbers of proliferating neuronal progenitors in each cluster, we quantified the number of clusters of PCNA-positive cells, with the assumption that a cluster of proliferating neuronal progenitor cells was derived from a single Müller glial cell. At $68 \mathrm{~h}$ of constant light, uninjected and $t n f \alpha 5$-mis control retinal sections contained $18.9 \pm 1.3$ and $18.1 \pm 1.4$ clusters of PCNA-positive cells relative to only $13.3 \pm$ 1.2 clusters in the $t n f \alpha$ morphants (Fig. 7J, $p<0.02$ ). This significantly reduced number of PCNA-positive cell clusters in the tnf $\alpha$ morphant relative to the controls demonstrated that TNF $\alpha$ expression in the Müller glia was necessary to increase the number of proliferating Müller glia in the light-damaged retina.

TNF $\alpha$ expression in the light-damaged retina is required for Stat 3 and Asclla expression

To determine whether TNF $\alpha$ signaling is required for the increased expression of the transcription factors Stat 3 or Asclla in the light-damaged retina (Fausett et al., 2008; Nelson et al., 2012), we immunolocalized Stat 3 and Asclla in $t n f \alpha$ 5-mis albino control retinas (Fig. $8 A, B$, respectively), and tnf $\alpha$ morphant albino retinas (Fig. $8 D, E$ ) after 36 h of constant intense light. Uninjected retinas contained $62.7 \pm 4.0$ Stat3-positive cells and $31.8 \pm 2.8$ Asclla-positive cells (Fig. $8 C$, blue bars). The $t n f \alpha$ 5-mis control retinas contained similar, high numbers of Stat3-positive and Asclla-positive INL cells (Fig. $8 A-C$, red bars). In contrast, tnf $\alpha$ morphant retinas contained only $14.7 \pm 4.6$ Stat 3 -positive and $9.3 \pm 3.0$ Asclla-positive cells (Fig. $8 C$, green bars, $D, E$ ), which were both significantly reduced in number relative to the $\operatorname{tnf} \alpha$ 5 -mis control retinas ( $p=0.003$ and 0.02 , respectively).

To confirm that TNF $\alpha$ is required for the expression of Stat3 and Asclla, we used quantitative real-time PCR (qRT-PCR) to examine the expression of the stat 3 and asclla transcripts in darkadapted albino zebrafish that were either uninjected or injected and electroporated with either the $\operatorname{tnf} \alpha$ 5-mis control morpholino or the $t n f \alpha$ morpholino. As expected (Kassen et al., 2007; 

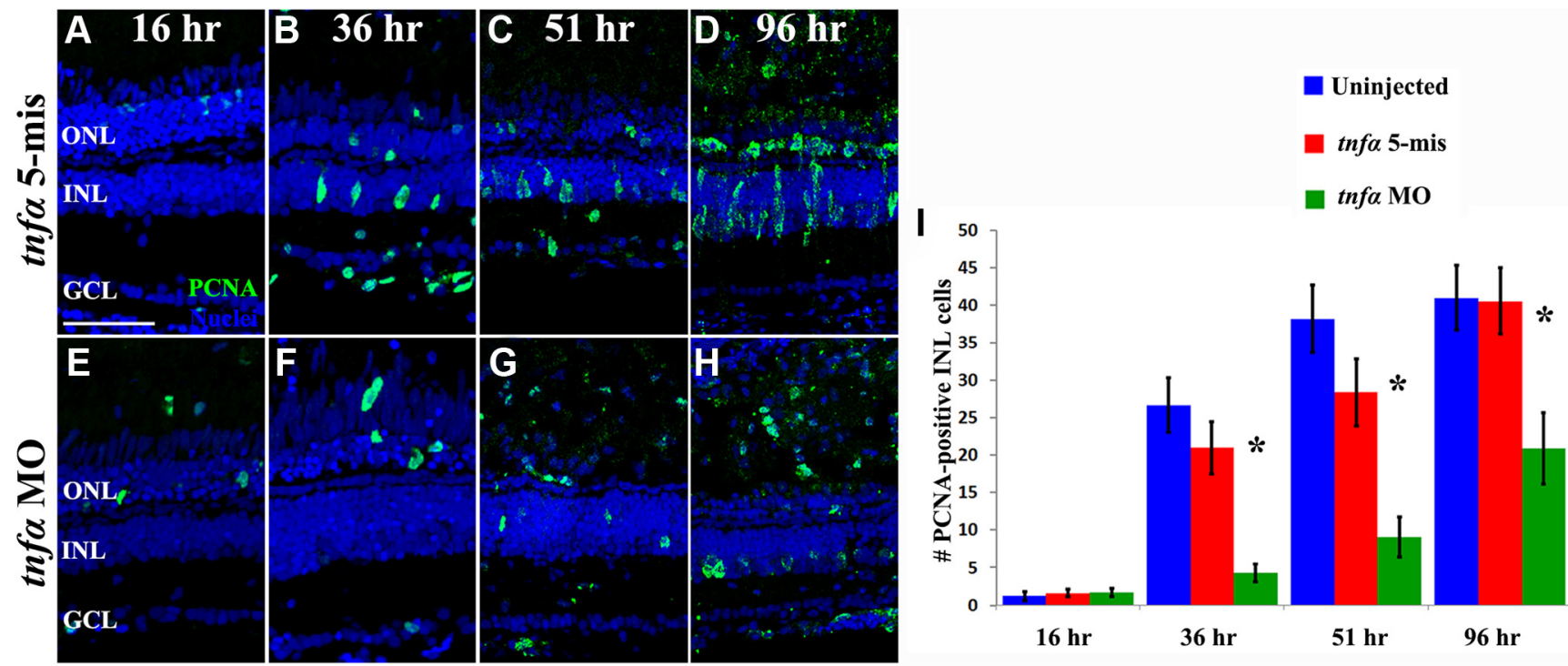

Figure 6. TNF $\alpha$ is required for Müller glial proliferation in the light-damaged retina. Dark-adapted albino zebrafish that were injected and electroporated with either the 5 -base mismatch control morpholino (tnf $\alpha$-mis, $\boldsymbol{A}-\boldsymbol{D})$ or the $\operatorname{tnf} \alpha$ morpholino $(\operatorname{tnf} \alpha \mathrm{MO}, \boldsymbol{E}-\boldsymbol{H})$ and exposed to constant intense light for either $16 \mathrm{~h}(\boldsymbol{A}, \boldsymbol{E}), 36 \mathrm{~h}(\boldsymbol{B}, \boldsymbol{F}), 51 \mathrm{~h}(\boldsymbol{C}, \boldsymbol{G})$, or $96 \mathrm{~h}(\boldsymbol{D}, \boldsymbol{H})$. The sections were immunolabeled with anti-PCNA to label the nuclei of dividing cells (green) and To-Pro-3 (blue) to label all nuclei. PCNA-positive cell counts were quantified in the INL $(I)$ from the uninjected and tnf $\alpha$ 5 -mis controls (blue and red bars, respectively) and the tnf $\alpha \mathrm{MO}$ (green bars). The tnf $\alpha$ morphants contained significantly fewer PCNA-positive INL cells relative to either control ( $p<0.0001, n=$ 10) at 36,51 , and $96 \mathrm{~h}$ of light treatment. Scale bar: (in $\boldsymbol{A}) \boldsymbol{A}-\boldsymbol{H}, 50 \mu \mathrm{m}$.

Bailey et al., 2010; Nelson et al., 2012), both stat3 and asclla RNA expression increased significantly within the first $16 \mathrm{~h}$ of constant light treatment in both the uninjected and tnf $\alpha$ 5-mis morpholino controls (Fig. 8F). However, both stat 3 and asclla RNA expression was significantly reduced in the tnf $\alpha$ morphant after $16 \mathrm{~h}$ of light relative to either the uninjected (Fig. $8 F, p=0.01$ and 0.04 , respectively) or $t n f \alpha 5$-mis control morphant (Fig. $8 F, p=$ 0.02 and 0.04 , respectively). While knockdown of TNF $\alpha$ expression could reduce the number of Müller glia (Thummel et al., 2008a), which in turn would reduce the overall expression of stat 3 and asclla in the light-damaged retina, we observed equal numbers of Müller glia after $48 \mathrm{~h}$ of constant light in the tnfo morphant and uninjected control retinas (data not shown). Additionally, we did not detect TUNEL-positive INL cells in either the tnf $\alpha$ morphant or uninjected control retinas after $48 \mathrm{~h}$ of constant light (data not shown). Together, these data indicated that TNF $\alpha$ signaling resides upstream and is required for both Stat 3 and Asclla expression during the earliest stages of retinal regeneration.

We previously described a model where the expression of Lin28a, Asclla, and Stat3 are all required in a subset of proliferating Müller glia to induce a different subset of Müller glia to re-enter the cell cycle (Nelson et al., 2012). Because Müller glial expression of TNF $\alpha$ appeared to be required to induce additional Müller glia to proliferate (Fig. 7), we examined if either Lin28a, Ascl1a, or Stat 3 are required for TNF $\alpha$ expression in the Müller glia. Dark-adapted albino zebrafish were injected and electroporated with either the Standard Control morpholino (S.C. MO, which is not complementary to any known sequence in the zebrafish genome), the tnf $\alpha$ morpholino, stat 3 morpholino, asclla morpholino, or the lin28a morpholino, and then exposed to constant intense light for $36 \mathrm{~h}$. The Standard Control morphant retinas exhibited TNF $\alpha$ expression in the OPL and the Müller glia in the INL (Fig. 9A, bracket and arrows, respectively). The tnf $\alpha$ morphant retinas lacked the TNF $\alpha$ immunostaining in both the OPL and INL (Fig. 9B). In contrast, the stat3 (Fig. 9C), asclla (Fig. 9D), and lin28a (Fig. 9E) morphant retinas possessed a significant amount of TNF $\alpha$ immu- nostaining in the OPL, but showed dramatically less TNF $\alpha$ expression in the Müller glia relative to the Standard Control morphant. Thus, Stat3, Asclla, and Lin28a expression are required for TNF $\alpha$ expression in the Müller glia, but not in either the OPL or NFL.

\section{Morpholino-mediated knockdown of TNF $\alpha$ does not block} rod and cone regeneration in the light-damaged retina

We examined the consequences of TNF $\alpha$ knockdown on Müller glia proliferation at 1 and $3 \mathrm{~d}$ following $96 \mathrm{~h}$ of constant light treatment. At $1 \mathrm{~d}$ after light treatment, the $\operatorname{tnf} \alpha$ morphant retinas contained a significantly greater number of PCNA-positive INL cells (52.8 \pm 4.3 ; Fig. $10 C, E$, green bars) relative to either the uninjected (33.1 \pm 8.1$)$ or tnf $\alpha$ 5-mismatch controls ( $26.0 \pm 4.5$; Fig. $10 A, E$, blue and red bars, respectively, $p<0.01$ ). Similarly, at $3 \mathrm{~d}$ after light treatment, the $\operatorname{tnf} \alpha$ morphant possessed significantly more PCNA-positive INL cells (95.4 \pm 7.2 ; Fig. 10D,E, green bars) relative to either uninjected $(42.3 \pm 4.5)$ or $\operatorname{tnf} \alpha$ 5-mismatch controls (41.4 \pm 6.0 ; Fig. $10 B, E$, blue and red bars, respectively). Thus, the tnf $\alpha$ morphant exhibited delayed Müller glia proliferation relative to controls.

We examined if the delayed Müller glia proliferation in the lightdamaged tnf $\alpha$ morphant still regenerated rod and cone photoreceptors. We confirmed the extent of rod and cone photoreceptor cell loss in the light-damaged tnf $\alpha$ morphants relative to the controls. After $96 \mathrm{~h}$ of constant light, uninjected (data not shown), tnfo 5-mismatch retinas (Fig. 11 A,E,I) and tnf $\alpha$ morphant retinas (Fig. $11 B, F, J)$ contained very low levels of Rhodopsin-, Blue opsin-, and UV opsin-expressing photoreceptors, suggesting a similar extent of photoreceptor cell loss in the tnf $\alpha$ morphant and the control retinas. However, the control retinas possessed a thicker and more densely packed ONL relative to the $\operatorname{tnf} \alpha$ morphant ONL. This is likely due to the $\operatorname{tnf} \alpha$ morphants failing to generate large numbers of progenitor cells that migrated to the ONL by $96 \mathrm{~h}$ of light treatment (Fig. 6).

We next examined the extent of photoreceptor cell regeneration $14 \mathrm{~d}$ after terminating $96 \mathrm{~h}$ of constant light. Uninjected (data not shown) and tnfo 5-mis retinas (Fig. 11C,G,K) contained high levels of Rhodopsin-, Blue opsin-, and UV opsin-labeled photoreceptor 

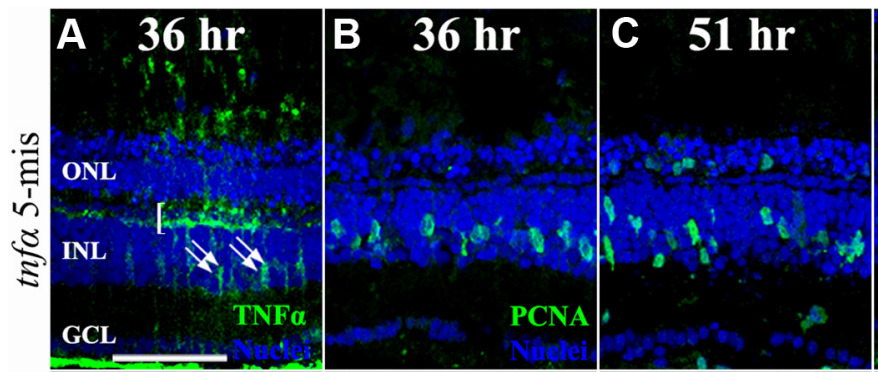

\section{D $68 \mathrm{hr}$}
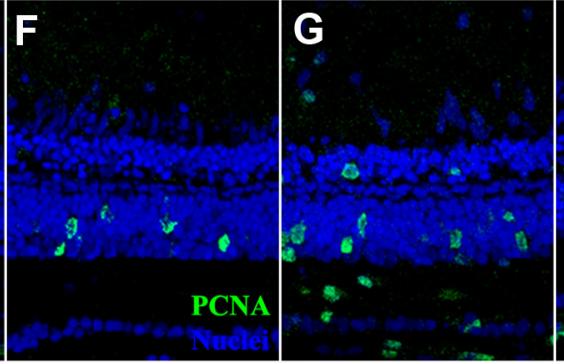

H

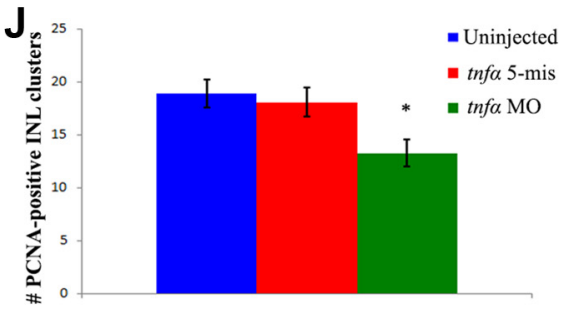

Figure 7. Müller glia-derived TNF $\alpha$ expression is required for inducing additional Müller glia to re-enter the cell cycle. Darkadapted albino zebrafish were exposed to constant intense light for $16 \mathrm{~h}$ and then intravitreally injected and electroporated with either lissamine-tagged tnf $\alpha$ 5-mismatch morpholinos (tnf $\alpha$ 5-mis, $\boldsymbol{A}-\boldsymbol{D}$ ) or translation-inhibiting tnf $\alpha$ morpholinos (tnf $\alpha \mathrm{M} 0$, $\boldsymbol{E}-\boldsymbol{H})$ before being placed back into intense light until $36 \mathrm{~h}(\boldsymbol{A}, \boldsymbol{B}, \boldsymbol{E}, \boldsymbol{F}), 51 \mathrm{~h}(\boldsymbol{C}, \boldsymbol{G})$, or $68 \mathrm{~h}$ of treatment $(\boldsymbol{D}, \boldsymbol{H})$. Retinal sections were immunolabeled with either anti-TNF $\alpha(\boldsymbol{A}, \boldsymbol{E}$, green) or anti-PCNA ( $\boldsymbol{B}-\boldsymbol{D}, \boldsymbol{F}-\boldsymbol{H}$, green) and To-Pro-3 ( $\boldsymbol{A}-\boldsymbol{H}$, blue) to label nuclei. Injection and electroporation of the tnf $\alpha \mathrm{M} 0$ after $16 \mathrm{~h}$ of constant light efficiently knocked down TNF $\alpha$ expression in the OPL, INL, and NFL relative to the control. INL PCNA-positive cell were quantified $(I)$ from uninjected and tnf $\alpha 5$-mismatch control eyes (blue and red bars, respectively) and the tnf $\alpha$ morphant (green bars) at 36,51, and $68 \mathrm{~h}$ of light. The number of PCNA-labeled INL cells was significantly decreased in the tnf $\alpha$ morphant relative to both controls $(p<0.005, n=10)$ at all three time points. PCNApositive INL cell clusters were quantified $(\boldsymbol{J})$ from uninjected and thf $\alpha$ 5-mismatch control eyes (blue and red bars, respectively) and the tnf $\alpha$ morphant (green bars) at $68 \mathrm{~h}$ of light. The number of PCNA-labeled cell clusters was significantly decreased in the tnf $\alpha$ morphant relative to both controls ( $p<0.05, n=10$ ). Scale bar: (in $\boldsymbol{A}) \boldsymbol{A}-\boldsymbol{H}, 50 \mu \mathrm{m}$.

outer segments, indicating that rods, double cones, and UV cones had regenerated, respectively. The $t n f \alpha$ morphants possessed similar levels of double and UV cone outer segments relative to the controls (Fig. $11 H, L$, respectively), suggesting that cones had regenerated in the $t n f \alpha$ morphant. The $t n f \alpha$ morphants also possessed rhodopsinpositive rod outer segments, although they were shorter than the controls, suggesting that rod photoreceptor regeneration was delayed in the $\operatorname{tnf} \alpha$ morphant relative to the controls.

\section{TNF $\alpha$ is required for Müller glia proliferation in the ouabain-damaged retina}

We were interested if TNF $\alpha$ is expressed in only dying photoreceptors or if it is expressed in other dying retinal neurons as a prerequisite to induce Müller glia to re-enter the cell cycle. This would reveal whether $\mathrm{TNF} \alpha$ served as a specific signal to Müller glia to proliferate and regenerate only photoreceptor cells or if TNF $\alpha$ was a general signal to induce Müller glia to re-enter the cell cycle to regenerate retinal neuronal classes in addition to photoreceptors.

To distinguish between these two possibilities, we intravitreally injected a low concentration of ouabain to induce the death of ganglion, amacrine, and bipolar cells without causing any significant photoreceptor cell death (Fimbel et al., 2007). We immu- nolocalized TNF $\alpha$ expression at 0,1 , and $3 \mathrm{~d}$ post ouabain injection. In the undamaged retina, TNF $\alpha$ expression was weakly detected in the OPL and NFL (Fig. $12 A, B)$. At $1 \mathrm{~d}$ post ouabain injection, TNF $\alpha$ expression increased dramatically in the OPL, the NFL, and cells within the GCL and INL that were morphologically different from Müller glia (Fig. 12C,D, bar, asterisks, and arrowheads, respectively). Some of the TNF $\alpha$-positive cells in the INL and GCL coexpressed $\mathrm{HuC} / \mathrm{D}$ (Fig. 12D, red), demonstrating that they were amacrine and ganglion cells, respectively. Colocalization of $\mathrm{TNF} \alpha$ and TUNEL at $1 \mathrm{~d}$ post ouabain injection confirmed that TUNEL-positive ganglion cells and INL cells expressed TNF $\alpha$ (Fig. $12 E)$. At $3 \mathrm{~d}$ postinjection, the TNF $\alpha$ expression persisted in the OPL, INL, and GCL, but was now also detected within INL cells that were morphologically similar to Müller glia (Fig. 12G,H, arrows). Immunolocalization of TNF $\alpha$ in the ouabaindamaged $\operatorname{Tg}(g f a p$ :EGFP)nt11 transgenic retinas confirmed that many TNF $\alpha$-positive INL cells were Müller glia at $3 \mathrm{~d}$ after ouabain injection (Fig. 12I, J, arrows). Thus, ouabain-induced inner retinal damage stimulated $\mathrm{TNF} \alpha$ expression in apoptotic inner retinal neurons and subsequently the Müller glia.

To determine whether TNF $\alpha$ expression was required to induce Müller glia to re-enter the cell cycle in the ouabaindamaged retina, in the absence of significant photoreceptor cell damage (Fimbel et al., 2007), we intravitreally injected a final concentration of $2 \mu \mathrm{M}$ ouabain immediately following injecting nothing (Fig. 13A, Ouabain control) or injecting and electroporating either the $t n f \alpha 5$-mismatch control morpholino or the $t n f \alpha$ morpholino (Fig. $13 B, C$ ). At $3 \mathrm{~d}$ postinjection, both the uninjected and the $\operatorname{tnf} \alpha$ 5-mis control retinas contained similar numbers of PCNA-positive cells, $57.0 \pm 4.9$ and $52.8 \pm 4.0$, respectively (Fig. 13A, B,D; blue and red bars, respectively). In contrast, the $\operatorname{tnf} \alpha$ morphants contained significantly fewer PCNApositive cells, $29.9 \pm 6.5$ (Fig. $13 C, D$, green bar, $p=0.008$ relative to the $t n f \alpha$ 5-mis control). These results demonstrated that both apoptotic photoreceptors and INL/GCL neurons expressed TNF $\alpha$ that was required for inducing maximal numbers of proliferating Müller glia. Furthermore, this demonstrates that TNF $\alpha$ plays a general role to initiate Müller glia proliferation in response to the loss of any neuronal cell-type within the zebrafish retina.

\section{Human-recombinant HB-EGF is not sufficient to induce Müller glia proliferation}

Because HB-EGF was previously shown to be both necessary and sufficient for Müller glia proliferation (Wan et al., 2012), we sought to examine if HB-EGF was sufficient to induce TNF $\alpha$ expression in either neurons or Müller glia. We intravitreally injected either human recombinant HB-EGF protein (Fig. $14 C, D)$, which was sufficient to stimulate Müller glia prolifera- 
tion (Wan et al., 2012), or vehicle (1X PBS, $0.1 \%$ BSA; Fig. 14A, $B$ ). The eyes were collected at either 1 (Fig. 14A, C) and 3 (Fig. $14 B, D$ ) dpi and immunolabeled for PCNA to identify proliferating Müller glia (Fig. 14A-D). PCNA-positive INL cell counts were acquired from optic nerve containing sections to ensure consistency between HB-EGF-injected eyes and vehicle controls. HB-EGF-injected retinas contained $1.3 \pm 0.3$ and $1.6 \pm 0.5$ PCNApositive INL cells at 1 and $3 \mathrm{dpi}$, respectively, which was not significantly different relative to vehicle-injected retinas at 1 and $3 \mathrm{dpi}(1.2 \pm 0.3$ and $1.5 \pm 0.4$ PCNA-positive cells, respectively; $p=$ 0.84 and 0.87 , respectively). The number of PCNA-expressing cells in the ONL of HB-EGF-injected eyes (15.0 \pm 2.4 and $20.3 \pm 1.7$ at 1 and 3 dpi, respectively) was also not statistically different relative to vehicle-injected eyes at 1 and $3 \mathrm{dpi}$ $(17.1 \pm 3.0$ and $17.3 \pm 2.8$, respectively; $p=0.60$ and 0.37 , respectively).

Because HB-EGF was not sufficient to induce Müller glia proliferation in the undamaged retina, we examined the expression pattern of $h b$-egfa mRNA in the lightdamaged retina. Using RT-PCR to examine the temporal expression of $h b$-egfa, we found that the mRNA increased in expression from 0 to $16 \mathrm{~h}$ of light and then again at $36 \mathrm{~h}$ of light (Fig. 14E). This is in agreement with Wan et al. (2012), which described a rapid increase in $h b$-egfa expression after the retinal puncture wound. Using in situ hybridization, a low level of $h b$-egfa was detected in both the ONL and INL in the undamaged retina (Fig. 14F). At $36 \mathrm{~h}$ of constant light, there was a dramatic increase of $h b$-egfa expression in both the ONL and INL (Fig. 14G). Several of the hb-egfapositive cells in the INL possessed a morphology that is consistent with Müller glia (Fig. 14G, arrows), while the remaining labeled cells likely represent proliferating Müller glia that are migrating to the ONL (Figure 14G, arrowheads; Qin et al., 2009; Wan et al., 2012). At $51 \mathrm{~h}$, the hb-egfa expression localized primarily to the INL and possessed a Müller glial morphology (Fig. $14 H$, arrows). Surprisingly, morpholinomediated knockdown of HB-EGFa expression in the light-damaged retina did not significantly reduce the number of proliferating Müller glia relative to either the uninjected or Standard Control morphant light-damaged retinas (data not shown). Together, hb-egfa expression increases in the Müller glia, but it does not appear to play a significant role in Müller glia proliferation in the lightdamaged retina.

\section{Discussion}

TNF $\alpha$ represents the first and only signal identified that is produced by apoptotic retinal neurons to induce Müller glia prolif-
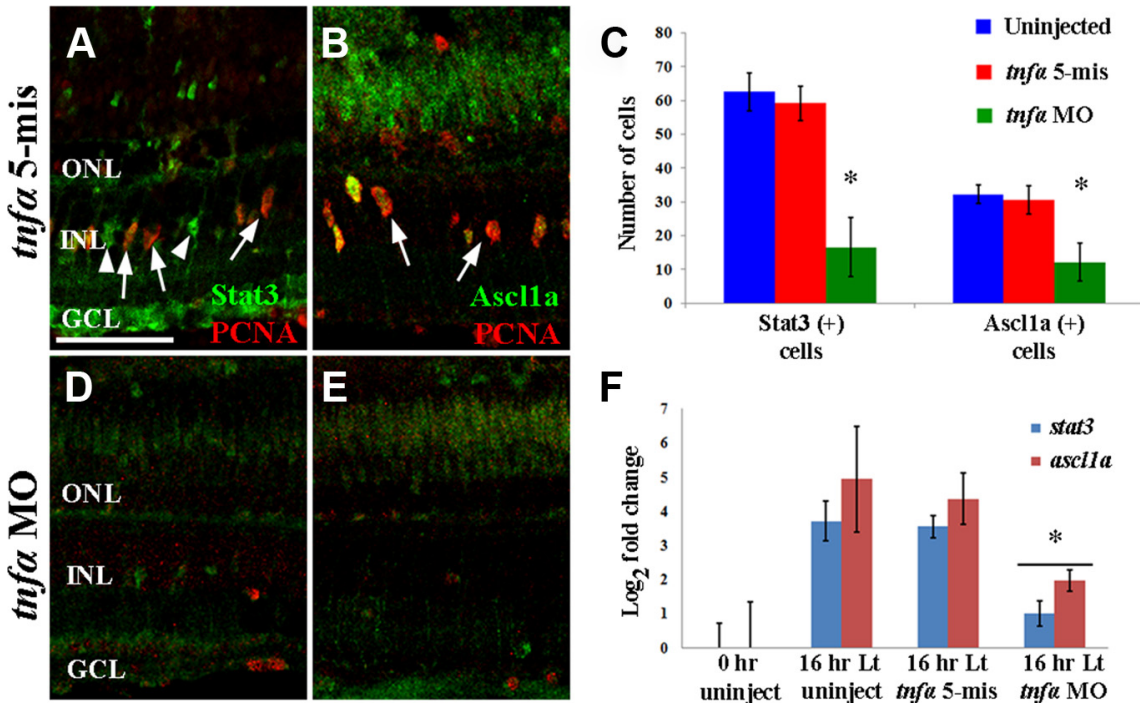

Figure 8. TNF $\alpha$ expression is required for Stat3 and Ascl1a expression. Dark-adapted albino zebrafish retinas were intravitreally injected and electroporated with either the lissamine-tagged tnf $\alpha$ 5-mismatch morpholino (tnf $\alpha$ 5-mis, $\boldsymbol{A}, \boldsymbol{B}$ ) or translationinhibiting tnf $\alpha$ morpholino (tnf $\alpha \mathrm{MO}, \boldsymbol{D}, \boldsymbol{E})$ and then exposed to constant intense light for $36 \mathrm{~h}$. Retinal cryosections were immunolabeled for PCNA ( $\boldsymbol{A}, \boldsymbol{B}, \boldsymbol{D}, \boldsymbol{E}$; red) and either Stat3 ( $\boldsymbol{A}, \boldsymbol{D}$; green) or Ascl1a ( $\boldsymbol{B}, \boldsymbol{E}$; green) and Stat3- and Ascl1a-labeled cells were quantified across a $350 \mu \mathrm{m}$ region of the central dorsal retina (C). Some Stat3-labeled cells were PCNA positive and others were PCNA negative in the control ( $\boldsymbol{A}$, arrows and arrowheads, respectively). In contrast, all the Ascl1a-labeled cells coexpressed PCNA in the control $(\boldsymbol{B})$. Relative to both controls ( $\boldsymbol{C}$; blue and red bars), the tnf $\alpha$ morphant (green bars) possessed significantly fewer Stat3-positive cells and Ascl1a-labeled cells than either the uninjected or $\operatorname{tnf} \alpha 5$-mis controls $(p<0.05, n=10)$. Total RNA was isolated from dorsal retinas of dark-adapted albino fish that were not light damaged ( 0 h uninject), placed in constant light for $16 \mathrm{~h}$ (16 h Lt uninject), and fish that were intravitreally injected and electroporated with either the lissamine-tagged tnf $\alpha$ 5-mismatch morpholinos (tnf $\alpha$ 5-mis) or translation-inhibiting tnf $\alpha$ morpholinos (tnf $\alpha \mathrm{M} 0$ ) and light damaged for $16 \mathrm{~h}$. The $\log _{2}$ of the mean expression for stat 3 and ascl $1 a$ determined by qRT-PCR ( $\boldsymbol{H}$; blue and red bars, respectively; $n=3)$ was plotted. The stat 3 and ascl1 $1 a$ expression was significantly increased in both $16 \mathrm{~h}$ light-damaged control groups relative to the undamaged $0 \mathrm{~h}$ control $(p<0.05)$. Additionally, the expression of both stat3 and ascl1a was significantly reduced in the light-damaged tnf $\alpha$ morphants relative to either of the light-damaged controls ( $p<0.04$ ). Scale bar: (in $\boldsymbol{A}), \boldsymbol{A}, \boldsymbol{B}, \boldsymbol{D}, \boldsymbol{E}, 50 \mu \mathrm{m}$.

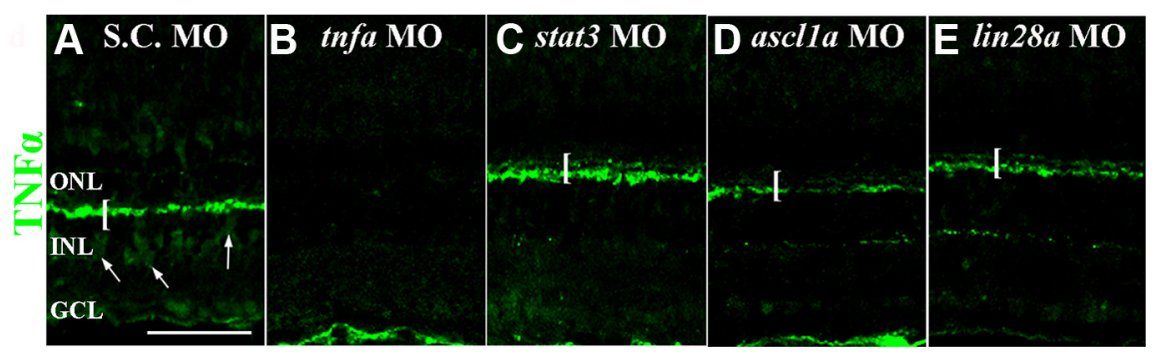

Figure 9. Müller glia, but not outer plexiform, expression of TNF $\alpha$ requires Stat3, Ascl1a, and Lin28a. Dark-adapted adult albino zebrafish retinas were intravitreally injected and electroporated with either standard control morpholino (S.C. M0, A), tnf $\alpha$ morpholino (tnf $\alpha \mathrm{M0}, \mathbf{B}$ ), stat3 morpholino (stat3 M0, C), ascl1 a morpholino (ascl1a M0, D), or lin28a morpholino (lin28a M0, E) and then exposed to constant intense light for $36 \mathrm{~h}$. Retinal cryosections were immunolabeled for TNF $\alpha$ (green). Robust TNF $\alpha$ expression was observed in the OPL (bracket) and INL (arrows) of the S.C. morphant control retina (A). Both OPL and INL TNF $\alpha$ labeling was nearly abolished in the tnf $\alpha$ morphant retinas $(\boldsymbol{B})$. The OPL immunolabeling was unaffected in the stat3, ascl1a, and lin28a morphants ( $\boldsymbol{C}-\boldsymbol{E}$, brackets), but the INL expression of TNF $\alpha$ was nearly absent. Scale bar: (in $\boldsymbol{A}) \boldsymbol{A}-\boldsymbol{E}, 50 \mu \mathrm{m}$.

eration at the initiation of zebrafish retinal regeneration. Photoreceptor-derived TNF $\alpha$ was also required for Asclla and Stat 3 expression in the Müller glia. In contrast, Lin28a, Asclla, and Stat 3 are all necessary for TNF $\alpha$ expression in the Müller glia and the maximal number of proliferating Müller glia in the lightdamaged retina.

Zebrafish Müller glia respond to retinal damage by reentering the cell cycle (Yurco and Cameron, 2005; Fausett and Goldman, 2006; Kassen et al, 2007; Fimbel et al., 2007; Thummel et al., 2008a,b; Bailey et al., 2010; Craig et al., 2010). We hypothesized that apoptotic neurons produced a signal that induced 

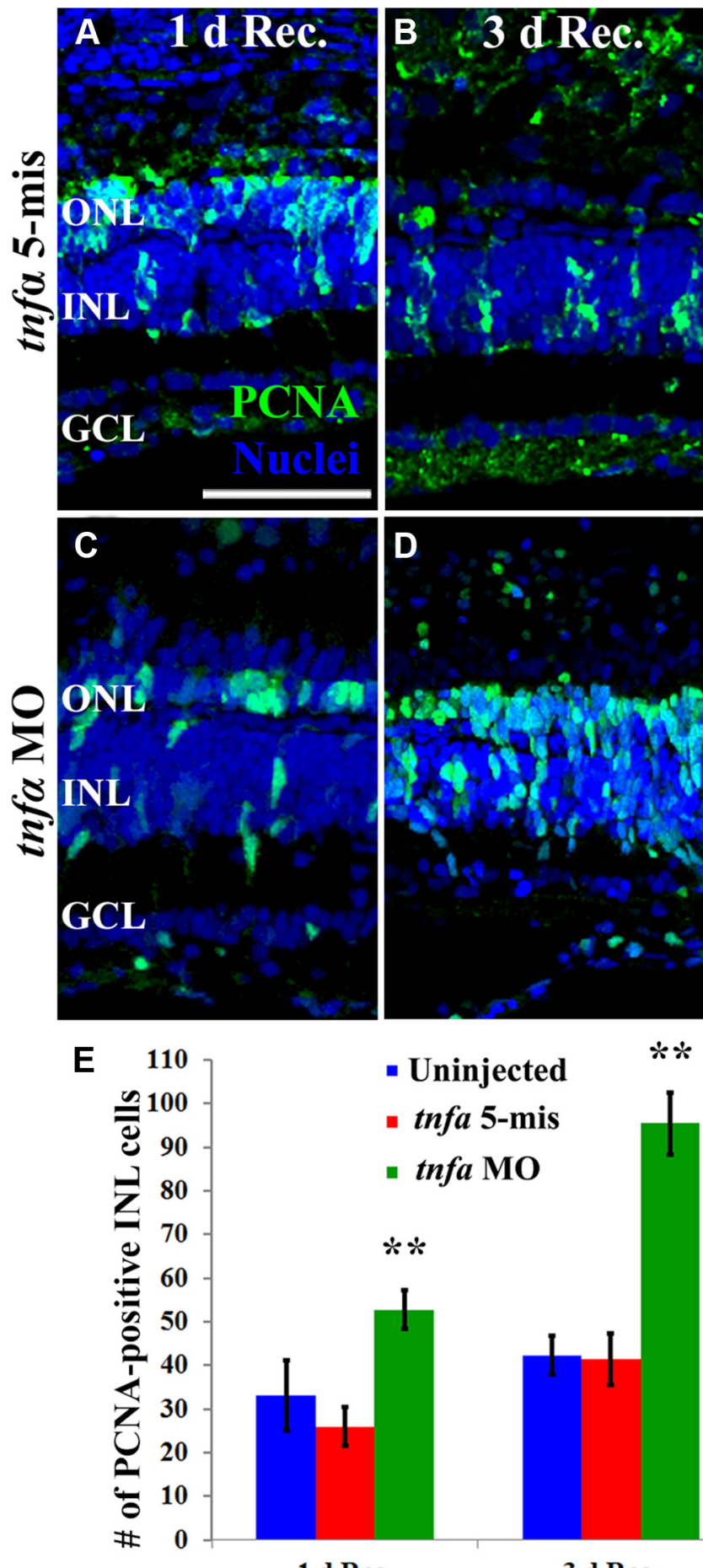

- Uninjected

- tnfa 5-mis

- tnfa MO

$* *$

3 d Rec.

Figure 10. TNF $\alpha$ knockdown delays Müller glial proliferation in the light-damaged retina. Dark-adapted albino zebrafish that were injected and electroporated with either the 5-base mismatch control morpholino $(\operatorname{tnf} \alpha 5$-mis, $A, B)$ or the $\operatorname{tnf} \alpha$ morpholino $(\operatorname{tnf} \alpha \mathrm{MO}, C, D)$ and exposed to constant intense light for $96 \mathrm{~h}$ followed by either $1(\boldsymbol{A}, \boldsymbol{C})$ or $3 \mathrm{~d}$ of recovery $(\boldsymbol{B}, \boldsymbol{D})$. The sections were immunolabeled with anti-PCNA to label the nuclei of dividing cells (green) and To-Pro-3 (blue) to label all nuclei. PCNA-positive INL cell counts were quantified $(\boldsymbol{E})$ from the uninjected and tnf $\alpha$-mis controls (blue and red bars, respectively) and the tnf $\alpha$ MO (green bars). The $\operatorname{tnf} \alpha$ morphants contained significantly more PCNA-positive INL cells relative to either control $(p<0.001, n=10)$ at both 1 and 3 d recovery from 96 h of light treatment. Scale bar: (in $\boldsymbol{A}) \boldsymbol{A}-\boldsymbol{D}, 50 \mu \mathrm{m}$.
Müller glia proliferation. Intravitreal injection of light-damaged retinal homogenates collected after $16 \mathrm{~h}$ of constant light was sufficient to induce significantly more proliferating Müller glia, without causing neuronal cell death, relative to undamaged retinal homogenates. While the light-damaged homogenate induced far fewer proliferating Müller glia relative to the light-damaged retina, this could be due to diffusion of the homogenate's signaling molecules into the vitreous, reduced penetration of the signals through the retina, or degradation of the signaling molecules. Additionally, the reduced number of proliferating Müller glia may be due to the absence of any cell death and the retention of cell-cell contacts.

Immunolocalization and in situ hybridization confirmed that $\mathrm{TNF} \alpha$ expression increased in apoptotic photoreceptors and later in Müller glia. Knockdown of TNF $\alpha$ expression before the start of the light treatment dramatically reduced $\mathrm{TNF} \alpha$ expression throughout the retina and significantly reduced the number of PCNA-labeled INL cells by $82 \%$ relative to control retinas. In contrast, knockdown of TNF $\alpha$ expression after $16 \mathrm{~h}$ of light treatment inhibited TNF $\alpha$ expression in the Müller glia, but not in photoreceptor cells, the OPL, and NFL. This latter knockdown significantly reduced the number of PCNA-labeled INL cells by $40 \%$ relative to controls, similar to the $50 \%$ reduction of proliferating Müller glia in the stat 3 morphant retina relative to controls (Nelson et al., 2012). This suggests that Müller glial-derived TNF $\alpha$ and Stat 3 function at a similar point that induces maximal numbers of proliferating Müller glia. Additionally, knockdown of TNF $\alpha$ expression in only the Müller glia yielded fewer PCNAlabeled INL cell clusters, rather than fewer neuronal progenitor cells per cluster. Because each proliferating INL cell cluster likely represents the neuronal progenitor cells derived from a single proliferating Müller glial cell, the reduced number of proliferating clusters likely corresponds to fewer Müller glia re-entering the cell cycle. Thus, apoptotic photoreceptor-derived TNF $\alpha$ expression induced an initial population of Müller glia to re-enter the cell cycle, while the Müller glia-derived TNF $\alpha$ expression induced a secondary population of proliferating Müller glia.

These two populations of proliferating Müller glia are consistent with a model of dying photoreceptors activating a signaling cascade (Lin28a activating Stat3 through Asclla) within a subset of Müller glia (Primary Proliferating Müller glia, PPMg; Fig. 14; Ramachandran et al., 2010; Nelson et al., 2012). Lin28a and Ascl1a, but not Stat3, are required for PPMg proliferation; however, all three are necessary to stimulate a subsequent subset of Müller glia to proliferate (Secondary Proliferating Müller glia, $\mathrm{SPMg}$ ). This study demonstrates that TNF $\alpha$ is a signal produced by dying neurons that is necessary for expressing Stat 3 and Asclla in Müller glia, as well as Müller glia proliferation (Fig. 15). Additionally, knockdown of Lin28a, Asclla, or Stat3 dramatically reduced TNF $\alpha$ expression only in the Müller glia, suggesting that $\mathrm{TNF} \alpha$ is a signal produced by the PPMg to induce the SPMg to proliferate in a Stat3-dependent manner. Consistent with that hypothesis, knockdown of TNF $\alpha$ expression after $16 \mathrm{~h}$ of light significantly reduced the number of proliferating Müller glia or clusters of proliferating INL cells. These data indicate that TNF $\alpha$ is required for Müller glia to become responding progenitor cells, which enter the cell cycle, but not for the subsequent proliferation and expansion of the retinal progenitors.

While knockdown of TNF $\alpha$ expression significantly reduced the number of proliferating Müller glia during the light treatment, the number eventually recovered and the rods and cones regenerated (Fig. 11). This suggests that TNF $\alpha$ expression may increase after the transient morpholino-mediated knockdown 


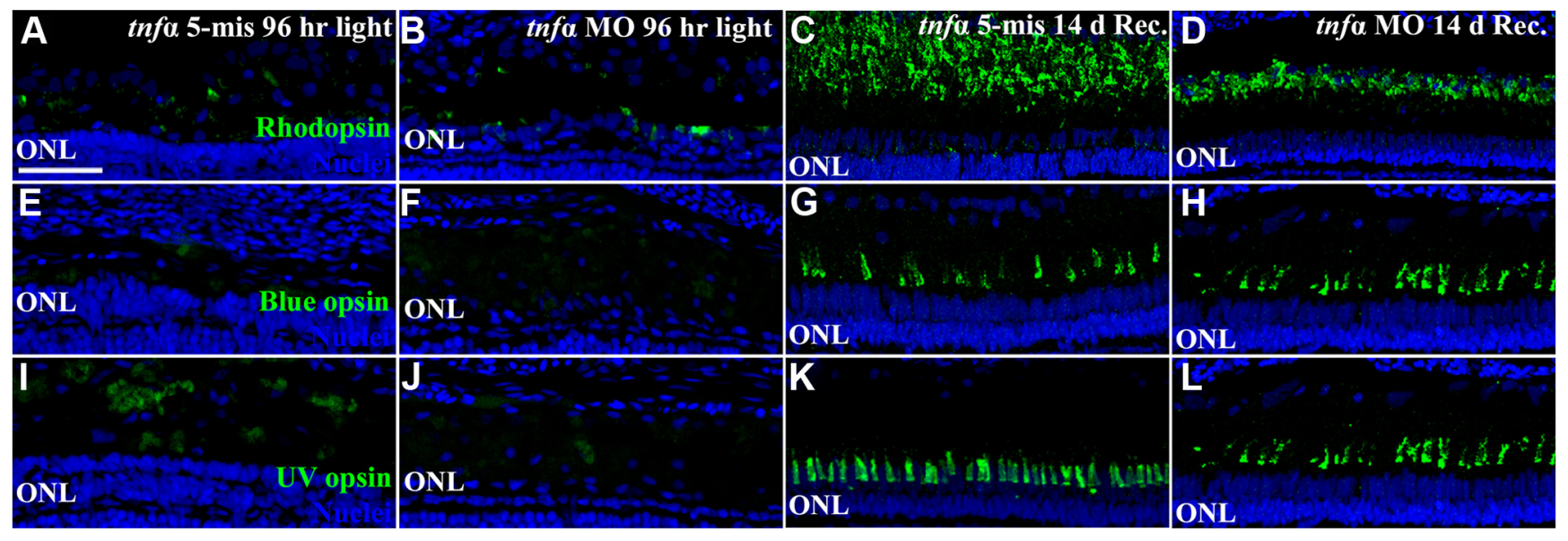

Figure 11. Aberrant rod photoreceptor regeneration following TNF $\alpha$ knockdown. Dark-adapted albino zebrafish that were injected and electroporated with either the 5 -base mismatch control morpholino

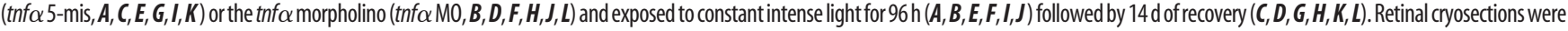
immunolabeled with either anti-Rhodopsin ( $A-D$, green), anti-Blue opsin $(\boldsymbol{E}-\boldsymbol{H}$, green), or anti-UV opsin (I-L, green) to label rods, double cones, and UV cones, respectively, and To-Pro-3 (blue) to label all nuclei. At $96 \mathrm{~h}$ of treatment, thf $\alpha$ morphants and controls contained low levels of opsin-positive photoreceptors $(A, B, E, F, I, J$, green). Following $14 \mathrm{~d}$ recovery, high levels of opsin expression were observed in tnf $\alpha$ 5-mis and tnf $\alpha$ morphants. However, the rod outer segment (ROS) length in tnf $\alpha$ morphants was decreased relative to the control. Scale bar: (in $A$ ) $A-L, 50 \mu \mathrm{m}$.

\section{Control}
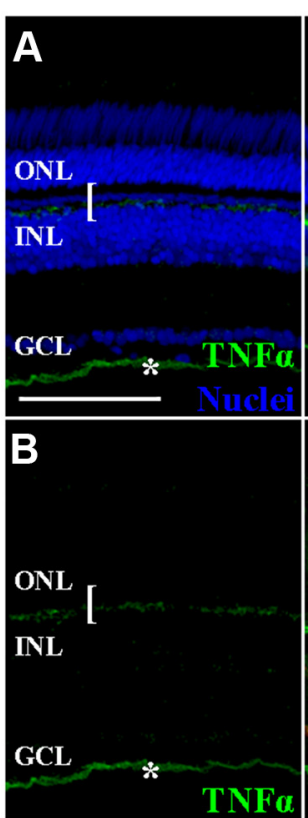

1 dpi
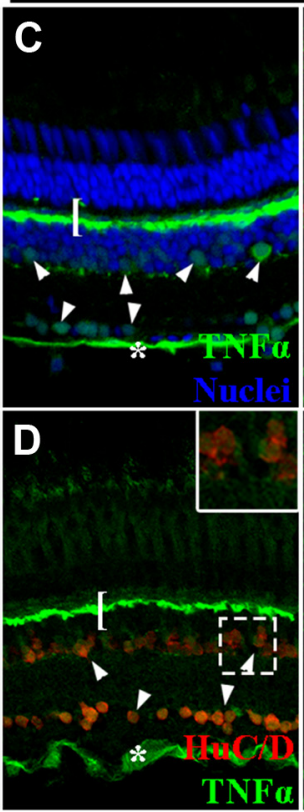
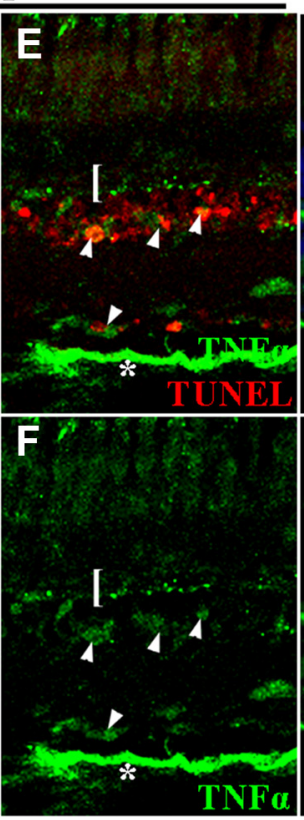

3 dpi

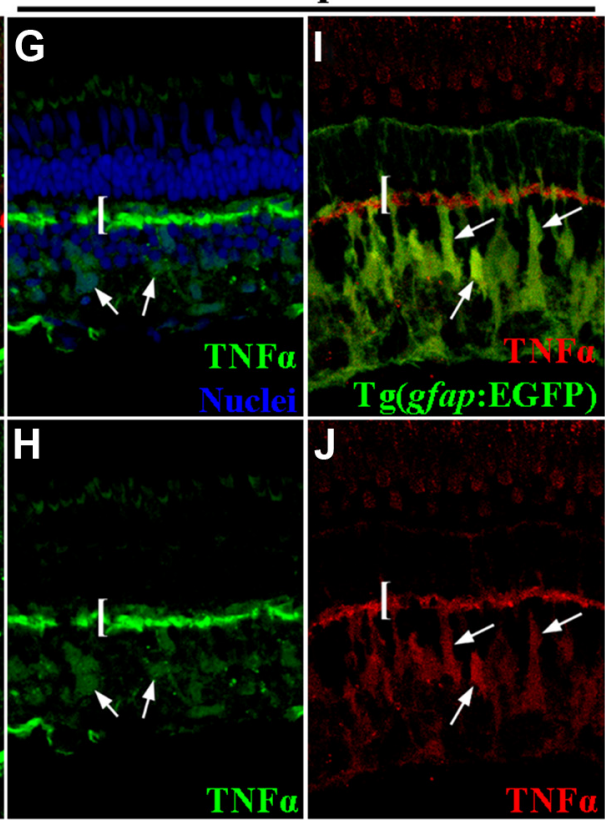

Figure 12. TNF $\alpha$ expression increased in apoptotic inner retinal neurons and Müller glia in the ouabain-damaged retina. Adult zebrafish were either uninjected $(\boldsymbol{A}, \boldsymbol{B})$ or intravitreally injected with ouabain to a final concentration of $2 \mu \mathrm{M}(\boldsymbol{C}-\boldsymbol{J})$. At $1(\boldsymbol{C}-\boldsymbol{F})$ and $3 \mathrm{~d}(\mathbf{G}-\boldsymbol{J})$ after ouabain injection, TNF $\alpha(\boldsymbol{A}-\boldsymbol{H}$, green; $\boldsymbol{I}, \boldsymbol{J}$, red) expression was immunolabeled with either HuC/D (D, red), TUNEL $(\boldsymbol{E}$, red), EGFP in the Tg (gfap:EGFP)nt11 transgenic line (I, green) or To-Pro-3 to identify the nuclear layers ( $\boldsymbol{A}, \boldsymbol{C}, \mathbf{G}$, blue). TNF $\alpha$ was observed in undamaged control retinas in the OPL and NFL (bracket and asterisk, respectively). At 1 dpi, TNF $\alpha$ expression increased in the OPL (bracket), NFL (asterisk), INL, and GCL (arrowheads). Some of the TNF $\alpha$-positive INL and GCL cells colabeled with HuC/D and TUNEL-positive nuclei ( $\boldsymbol{D}, \boldsymbol{E}$, respectively). At 3 dpi, increased TNF $\alpha$ expression persisted in the OPL (bracket) and the INL (arrows). The TNF $\alpha$-positive INL cells (red) colocalized with EGFP (green) in the $\operatorname{Tg}($ ffap:EGFP)nt11 transgenic retinas $(\boldsymbol{I}, \boldsymbol{J})$. The inset in $\boldsymbol{D}$ corresponds to the dashed box region. Scale bar: (in $\boldsymbol{A}) \boldsymbol{A}-\boldsymbol{J}, 50 \mu \mathrm{m}$.

ends. However, this TNF $\alpha$ expression would have to come from cells other than dying rods and cones, which are largely lost by 3 or $4 \mathrm{~d}$ of light damage. These TNF $\alpha$-expressing cells could be the few remaining photoreceptors or some other retinal cell type that produces sufficient TNF $\alpha$ to induce the delayed Müller glia proliferation response. Alternatively, a delayed signal other than $\mathrm{TNF} \alpha$ may induce Müller glia to re-enter the cell cycle, although the identity and source of this signal is unknown.

Morpholino-mediated knockdown of HB-EGF in puncturedamaged retinas and injecting human HB-EGF into undamaged eyes demonstrated that HB-EGF was both necessary and sufficient for Müller glia proliferation (Wan et al., 2012). Because $h b$-egf was expressed in Müller glia rather than damaged neurons (Wan et al., 2012; Fig. 14), it was unlikely to be required for TNF $\alpha$ expression in the dying neurons. To confirm this, we tested if HB-EGF-induced proliferating Müller glia in the undamaged retinas induced TNF $\alpha$ expression. However, we could not induce Müller glia proliferation by intravitreal injection of HB-EGF into undamaged retinas. There are several reasons why our results differed from those of Wan et al. (2012). We used a sapphire 

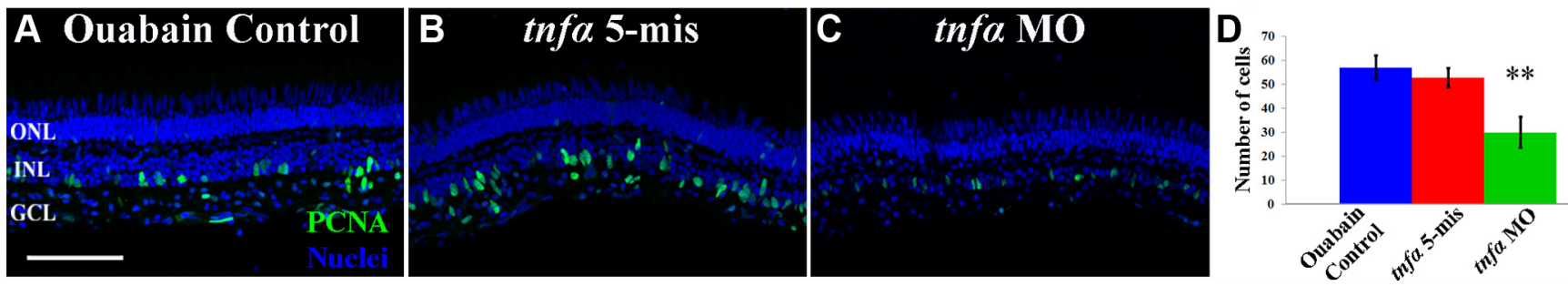

Figure 13. TNF $\alpha$ is required for the maximal number of Müller glia to re-enter the cell cycle in the ouabain-damaged retina. Adult zebrafish were either uninjected $(A)$ or intravitreally injected and electroporated with lissamine-tagged tnf $\alpha$ 5-mismatch morpholinos (tnf $\alpha$ 5-mis, $B$ ) or translation-inhibiting tnf $\alpha$ morpholinos $(\operatorname{tnf} \alpha \mathrm{MO}, \mathrm{C})$ and then intravitreally injected with ouabain to a final concentration of $2 \mu \mathrm{m}$. After $3 \mathrm{~d}$, retinal cryosections were immunolabeled for PCNA to detect the nuclei of dividing cells ( $\boldsymbol{A}-\boldsymbol{C}$, green) and stained with To-Pro-3 to identify the nuclear layers (blue). Both controls possessed significantly more PCNA-positive INL cells relative to the tnf $\alpha$ morphant at $3 \mathrm{dpi}$ ( $\boldsymbol{D}$; blue bar, red bar, green bar, respectively; $\boldsymbol{P}<0.01, n=10$ ). Scale bar: (in $\boldsymbol{A}) \boldsymbol{A}-\boldsymbol{C}, 50 \mu \mathrm{m}$
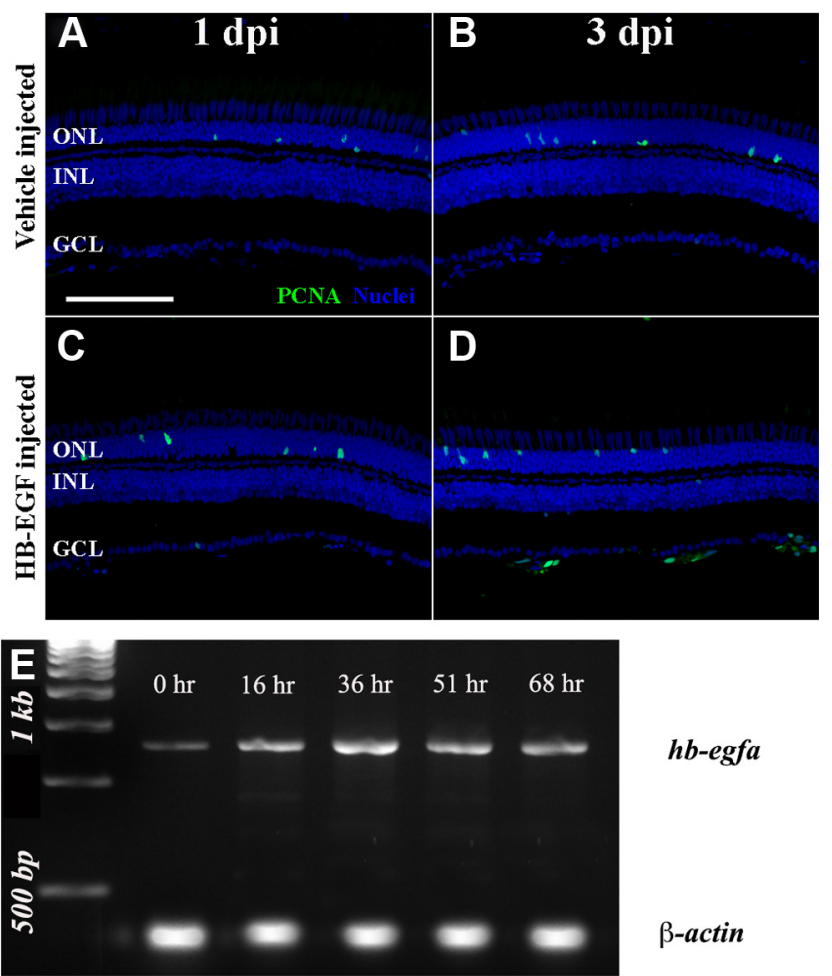

$h b-e g f a$
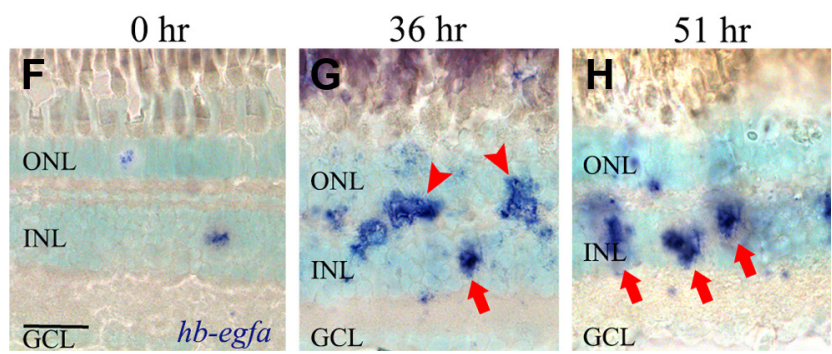

Figure 14. HB-EGF, which increases in expression in the Müller glia of the light-damaged retina, is not sufficient to induce Müller glia proliferation. Adult zebrafish were either injected with $1 \times P B S, 0.1 \% B S A$ vehicle solution $(A, B)$, or human recombinant $H B-E G F$ reconstituted in the vehicle solution $(\boldsymbol{C}, \boldsymbol{D})$. The eyes were collected at $1(\boldsymbol{A}, \boldsymbol{C})$ and $3(\boldsymbol{B}, \boldsymbol{D})$ dpi and processed for immunolabeling with anti-PCNA antibodies to detect the nuclei of dividing cells and To-Pro-3 nuclear stain ( $\boldsymbol{A}-\boldsymbol{D}$, green and blue, respectively). Dark-adapted albino zebrafish were placed in constant intense light for $0,16,36,51$, and $68 \mathrm{~h}$. Total RNA was isolated from the retinas and the $h b$-egfa and $\beta$-actin CDNAs were amplified by RT-PCR and analyzed by gel electrophoresis $(\boldsymbol{E})$. Dark-adapted albino zebrafish were exposed to constant intense light for $0 \mathrm{~h}(\boldsymbol{F}), 36 \mathrm{~h}(\boldsymbol{G})$, or $51 \mathrm{~h}(\boldsymbol{H}) \mathrm{h}$ and retinal cryosections were in situ hybridized with $h b$-egfa antisense riboprobe and counterstained with To-Pro-3. Low levels of $h b$-egfa were detected in the INL at $0 \mathrm{~h}$. In the light-damaged retinas, hb-egfa was detected in both the INL and the OPL (G, arrows and arrowheads, respectively). Scale bars: (in $\boldsymbol{A}) \boldsymbol{A}-\boldsymbol{D}, 50 \mu \mathrm{m}$; (in $\boldsymbol{F}) \boldsymbol{F}-\boldsymbol{H}, 20 \mu \mathrm{m}$.

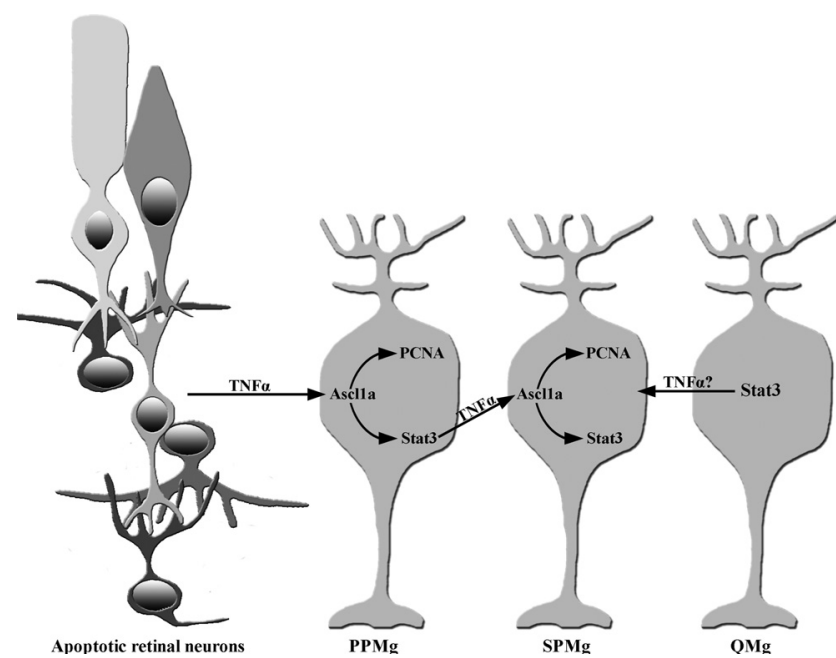

Figure 15. Model of TNF $\alpha$ signaling in the initiation and amplification of Müller glia proliferation during retinal regeneration. The light-damaged zebrafish retina possesses three classes of Müller glia: PPMg, SPMg, and Quiescent Müller glia (QMg). Apoptotic retinal neurons upregulate TNF $\alpha$ expression, which activates Ascl1a expression in the PPMg. Ascl1a drives the PPMg to re-enter the cell cycle (express PCNA) and induces Stat3 expression. Stat3 activates TNF $\alpha$ expression in the PPMg, which in turn, activates the expression of Ascl1a in the SPMg. The Ascl1a in the SPMg stimulates their proliferation and induces Stat3 expression. Thus, the PPMg proliferate in a TNF $\alpha$-dependent and Stat3-independent manner, while re-entry of the SPMg into the cell cycle requires Stat3- and Ascl1a-dependent TNF $\alpha$ expression in the PPMg. The QMg express Stat3, but do not express Ascl1a and do not proliferate. It remains unknown what activates Stat3 expression in the QMg.

blade to make an incision in the eye and inserted the needle through the opening to avoid the blunt force trauma of forcing the needle to penetrate into the eye. Further, we used a blunt needle rather than a beveled needle (Wan et al., 2012). The latter requires the needle to be inserted beyond the beveled edge to successfully inject the solution into the vitreous, which could damage the retina. Finally, injecting $2.0 \mu \mathrm{l}$ of $100 \mathrm{ng} / \mu \mathrm{l} \mathrm{HB}-\mathrm{EGF}$ (Wan et al., 2012), which is greater than the adult intravitreal volume (Fimbel et al., 2007), could result in high intraocular pressure and retinal damage. Because of this concern, we injected either $1.0 \mu \mathrm{l}$ of $200 \mathrm{ng} / \mu \mathrm{l}$ or $2.0 \mu \mathrm{l}$ of $100 \mathrm{ng} / \mu \mathrm{l}$ recombinant HB-EGF solution into the vitreous, which represents the same amount of recombinant HB-EGF described previously (200 ng; Wan et al., 2012). While injecting either HB-EGF volume resulted in some solution flowing out of the vitreous, neither volume induced significant numbers of proliferating Müller glia relative to controls. It is possible that injecting $2.0 \mu \mathrm{l}$ without the prior incision minimized the outflow and significantly increased the intraocular pressure. Some combination of these differences likely resulted in retinal stress or damage and HB-EGF could be 
sufficient to induce Müller glia proliferation under these conditions (Wan et al., 2012).

Because HB-EGF was not sufficient to induce Müller glia proliferation in the undamaged retina, we tested if it was necessary for Müller glia proliferation in the light-damaged retina to examine the relationship of HB-EGF and TNF $\alpha$. We injected and electroporated $h b$-egfa morpholinos into the retina immediately before starting the constant light treatment and found that it failed to significantly reduce the number of proliferating Müller glia relative to the Standard Control morphant retina (data not shown). While this difference between our result and Wan et al. (2012) could be due to the different damage models, HB-EGF is not required for inducing Müller glia proliferation in response to photoreceptor damage.

Ouabain damage to inner retinal neurons (Fimbel et al., 2007) increased TNF $\alpha$ expression in ganglion and INL cells at 1 dpi and in Müller glia by 3 dpi. Knockdown of TNF $\alpha$ before ouabain injection resulted in significantly fewer proliferating Müller glia relative to controls, suggesting that TNF $\alpha$ stimulates Müller glia to re-enter the cell cycle in response to retinal damage. However, numerous PCNA-positive Müller glia are present in the tnf $\alpha$ morphant retinas $3 \mathrm{~d}$ after ouabain injection (Fig. 13D). This suggests that at least one other unidentified TNF $\alpha$-independent signal induces Müller glia proliferation.

The mechanism by which TNF $\alpha$ induces Stat 3 expression in all Müller glia and Asclla in only proliferating Müller glia remains unclear (Kassen et al., 2007; Nelson et al., 2012). While $\mathrm{TNF} \alpha$ expression may stimulate Stat3 expression in all Müller glia, it is unclear why Asclla, which lies downstream of TNF $\alpha$ but upstream of Stat3 (Nelson et al., 2012), is expressed in only the proliferating Müller glia. It is possible that a regulatory loop exists between Stat3 and Asclla. Alternatively, TNF $\alpha$ may function with another retinal damage signal to differentially induce Asclla expression in only proliferating Müller glia and Stat 3 in all Müller glia. The identification of the TNF $\alpha$ receptor(s) and intracellular proteins regulated by the $\mathrm{TNF} \alpha$-bound receptor(s) will elucidate this mechanism. Ultimately, identifying the signaling pathways involved in Müller glia dedifferentiation and proliferation in the regenerating zebrafish model may reveal important mechanisms and therapeutic targets for enhancing mammalian retinal regeneration. Properly regulating these pathways may potentially induce Müller glia dedifferentiation and proliferation or promote exogenous pluripotent cells to actively and correctly regenerate lost neurons in the diseased or damaged human retina.

\section{References}

Bailey TJ, Fossum SL, Fimbel SM, Montgomery JE, Hyde DR (2010) The inhibitor of phagocytosis, O-phospho-L-serine, suppresses Müller glia proliferation and cone cell regeneration in the light-damaged zebrafish retina. Exp Eye Res 91:601-612. CrossRef Medline

Bernardos RL, Barthel LK, Meyers JR, Raymond PA (2007) Late-stage neuronal progenitors in the retina are radial Müller glia that function as retinal stem cells. J Neurosci 27:7028-7040. CrossRef Medline

Bringmann A, Pannicke T, Grosche J, Francke M, Wiedemann P, Skatchkov SN, Osborne NN, Reichenbach A (2006) Müller cells in the healthy and diseased retina. Prog Retin Eye Res 25:397-424. CrossRef Medline

Brockerhoff SE, Fadool JM (2011) Genetics of photoreceptor degeneration and regeneration in zebrafish. Cell Mol Life Sci 68:651-659. CrossRef Medline

Calinescu AA, Vihtelic TS, Hyde DR, Hitchcock PF (2009) Cellular expression of midkine-a and midkine-b during retinal development and photoreceptor regeneration in zebrafish. J Comp Neurol 514:1-10. CrossRef Medline

Chan FK, Lenardo MJ (2000) A crucial role for p80 TNF-R2 in amplifying p60 TNF-R1 apoptosis signals in T lymphocytes. Eur J Immunol 30:652-660. CrossRef Medline
Chen CF, Chen Y, Dai K, Chen PL, Riley DJ, Lee WH (1996) A new member of the hsp90 family of molecular chaperones interacts with the retinoblastoma protein during mitosis and after heat shock. Mol Cell Biol 16:46914699. Medline

Cheng B, Christakos S, Mattson MP (1994) Tumor necrosis factors protect neurons against metabolic-excitotoxic insults and promote maintenance of calcium homeostasis. Neuron 12:139-153. CrossRef Medline

Craig SE, Thummel R, Ahmed H, Vasta GR, Hyde DR, Hitchcock PF (2010) The zebrafish galectin Drgal1-L2 is expressed by proliferating Müller glia and photoreceptor progenitors and regulates the regeneration of rod photoreceptors. Invest Ophthalmol Vis Sci 51:3244-3252. CrossRef Medline

Do H, Pyo S, Sohn EH (2010) Suppression of iNOS expression by fucoidan is mediated by regulation of $\mathrm{p} 38 \mathrm{MAPK}$, JAK/STAT, AP- 1 and IRF- 1 , and depends on up-regulation of scavenger receptor Blexpression in TNF- $\alpha$ and IFN- $\gamma$-stimulated C6 glioma cells. J Nutr Biochem 21:671-679. CrossRef Medline

Fausett BV, Goldman D (2006) A role for alphal tubulin-expressing Müller glia in regeneration of the injured zebrafish retina. J Neurosci 26:6303-6313. CrossRef Medline

Fausett BV, Gumerson JD, Goldman D (2008) The proneural basic helixloop-helix gene asclla is required for retina regeneration. J Neurosci 28: 1109-1117. CrossRef Medline

Fimbel SM, Montgomery JE, Burket CT, Hyde DR (2007) Regeneration of inner retinal neurons after intravitreal injection of ouabain in zebrafish. J Neurosci 27:1712-1724. CrossRef Medline

Fischer AJ, Scott MA, Tuten W (2009) Mitogen-activated protein kinasesignaling stimulates Müller glia to proliferate in acutely damaged chicken retina. Glia 57:166-181. CrossRef Medline

Fleisch VC, Fraser B, Allison WT (2011) Investigating regeneration and functional integration of CNS neurons: lessons from zebrafish genetics and other fish species. Biochim Biophys Acta 1812:364-380. CrossRef Medline

Guo D, Dunbar JD, Yang CH, Pfeffer LM, Donner DB (1998) Induction of Jak/STAT signaling by activation of the type 1 TNF receptor. J Immunol 160:2742-2750. Medline

Gupta S (2001) Molecular steps of tumor necrosis factor receptor-mediated apoptosis. Curr Mol Med 1:317-324. CrossRef Medline

Hitchcock P, Kakuk-Atkins L (2004) The basic helix-loop-helix transcription factor neuroD is expressed in the rod lineage of the teleost retina. J Comp Neurol 477:108-117. CrossRef Medline

Karl MO, Reh TA (2010) Regenerative medicine for retinal diseases: activating endogenous repair mechanisms. Trends Mol Med 16:193-202. CrossRef Medline

Kassen SC, Ramanan V, Montgomery JE, T Burket C, Liu CG, Vihtelic TS, Hyde DR (2007) Time course analysis of gene expression during lightinduced photoreceptor cell death and regeneration in albino zebrafish. Dev Neurobiol 67:1009-1031. CrossRef Medline

Kassen SC, Thummel R, Campochiaro LA, Harding MJ, Bennett NA, Hyde DR (2009) CNTF induces photoreceptor neuroprotection and Müller glial cell proliferation through two different signaling pathways in the adult zebrafish retina. Exp Eye Res 88:1051-1064. CrossRef Medline

Kubota K, Inoue K, Hashimoto R, Kumamoto N, Kosuga A, Tatsumi M, Kamijima K, Kunugi H, Iwata N, Ozaki N, Takeda M, Tohyama M (2009) Tumor necrosis factor receptor-associated protein 1 regulates cell adhesion and synaptic morphology via modulation of $\mathrm{N}$-cadherin expression. J Neurochem 110:496-508. CrossRef Medline

Liu D, Hu J, Agorreta J, Cesario A, Zhang Y, Harris AL, Gatter K, Pezzella F (2010) Tumor necrosis factor receptor-associated protein 1 (TRAP1) regulates genes involved in cell cycle and metastases. Cancer Lett 296:194205. CrossRef Medline

MacEwan DJ (2002) TNF receptor subtype signalling: differences and cellular consequences. Cell Signal 14:477-492. CrossRef Medline

Matthews RP, Lorent K, Mañoral-Mobias R, Huang Y, Gong W, Murray IV, Blair IA, Pack M (2009) TNFalpha-dependent hepatic steatosis and liver degeneration caused by mutation of zebrafish S-adenosylhomocysteine hydrolase. Development 136:865-875. CrossRef Medline

Miscia S, Marchisio M, Grilli A, Di Valerio V, Centurione L, Sabatino G, Garaci F, Zauli G, Bonvini E, Di Baldassarre A (2002) Tumor necrosis factor alpha (TNF-alpha) activates Jak1/Stat3-Stat5B signaling through TNFR-1 in human B cells. Cell Growth Differ 13:13-18. Medline

Montgomery JE, Parsons MJ, Hyde DR (2010) A novel model of retinal ablation demonstrates that the origin of regenerated rod photoreceptors 
is dependent on the extent of damage. J Comp Neurol 518:800-814. CrossRef Medline

Mori T, Miyamoto T, Yoshida H, Asakawa M, Kawasumi M, Kobayashi T, Morioka H, Chiba K, Toyama Y, Yoshimura A (2011) IL- $1 \beta$ and TNF $\alpha$ initiated IL-6-STAT3 pathway is critical in mediating inflammatory cytokines and RANKL expression in inflammatory arthritis. Int Immunol 23:701-712. Medline

Nelson CM, Hyde DR (2012) Müller glia as a source of neuronal progenitor cells to regenerate the damaged zebrafish retina. Adv Exp Med Biol 723: 425-430. CrossRef Medline

Nelson CM, Gorsuch RA, Bailey TJ, Ackerman KM, Kassen SC, Hyde DR (2012) Stat3 defines three populations of Müller glia and is required for initiating maximal Müller glia proliferation in the regenerating zebrafish retina. J Comp Neurol 520:4294-4311. CrossRef Medline

Peng H, Whitney N, Wu Y, Tian C, Dou H, Zhou Y, Zheng J (2008) HIV-1infected and/or immune-activated macrophage-secreted TNF-alpha affects human fetal cortical neural progenitor cell proliferation and differentiation. Glia 56:903-916. CrossRef Medline

Peng H, Sun L, Jia B, Lan X, Zhu B, Wu Y, Zheng J (2011) HIV-1-infected and immune-activated macrophages induce astrocytic differentiation of human cortical neural progenitor cells via the STAT3 pathway. PLoS One 6:e19439. CrossRef Medline

Powell C, Elsaeidi F, Goldman D (2012) Injury-dependent Müller glia and ganglion cell reprogramming during tissue regeneration requires Apobec2a and Apobec2b. J Neurosci 32:1096-1109. CrossRef Medline

Qin Z, Barthel LK, Raymond PA (2009) Genetic evidence for shared mechanisms of epimorphic regeneration in zebrafish. Proc Natl Acad Sci U S A 106:9310-9315. CrossRef Medline

Ramachandran R, Fausett BV, Goldman D (2010) Asclla regulates Müller glia dedifferentiation and retinal regeneration through a Lin-28dependent, let-7 microRNA signalling pathway. Nat Cell Biol 12: 1101-1107. CrossRef Medline

Romanatto T, Cesquini M, Amaral ME, Roman EA, Moraes JC, Torsoni MA, Cruz-Neto AP, Velloso LA (2007) TNF-alpha acts in the hypothalamus inhibiting food intake and increasing the respiratory quotient-effects on leptin and insulin signaling pathways. Peptides 28:1050-1058. CrossRef Medline
Rowan S, Cepko CL (2004) Genetic analysis of the homeodomain transcription factor Chx10 in the retina using a novel multifunctional BAC transgenic mouse reporter. Dev Biol 271:388-402. CrossRef Medline

Tartaglia LA, Pennica D, Goeddel DV (1993) Ligand passing: the $75-\mathrm{kDa}$ tumor necrosis factor (TNF) receptor recruits TNF for signaling by the 55-kDa TNF receptor. J Biol Chem 268:18542-18548. Medline

Thomas JL, Nelson CM, Luo X, Hyde DR, Thummel R (2012) Characterization of multiple light damage paradigms reveals regional differences in photoreceptor loss. Exp Eye Res 97:105-116. CrossRef Medline

Thummel R, Kassen SC, Montgomery JE, Enright JM, Hyde DR (2008a) Inhibition of Müller glial cell division blocks regeneration of the lightdamaged zebrafish retina. Dev Neurobiol 68:392-408. CrossRef Medline

Thummel R, Kassen SC, Enright JM, Nelson CM, Montgomery JE, Hyde DR (2008b) Characterization of Müller glia and neuronal progenitors in zebrafish adult retinal regeneration. Exp Eye Res 87:433-444. CrossRef Medline

Thummel R, Enright JM, Kassen SC, Montgomery JE, Bailey TJ, Hyde DR (2010) Pax6a and Pax6b control novel regulatory points during neuronal progenitor cell proliferation in the regenerating zebrafish retina. Exp Eye Res 90:572-582. CrossRef Medline

Vihtelic TS, Hyde DR (2000) Light-induced rod and cone cell death and regeneration in the adult albino zebrafish (Danio rerio) retina. J Neurobiol 44:289-307. CrossRef Medline

Vihtelic TS, Doro CJ, Hyde DR (1999) Cloning and characterization of six zebrafish photoreceptor opsin cDNAs and immunolocalization of their corresponding proteins. Vis Neurosci 16:571-585. Medline

Vihtelic TS, Soverly JE, Kassen SC, Hyde DR (2006) Retinal regional differences in photoreceptor cell death and regeneration in light-lesioned albino zebrafish. Exp Eye Res 82:558 -575. CrossRef Medline

Wan J, Ramachandran R, Goldman D (2012) HB-EGF is necessary and sufficient for Müller glia dedifferentiation and retina regeneration. Dev Cell 22:334-347. CrossRef Medline

Wang Y, Wu TR, Cai S, Welte T, Chin YE (2000) Complex to inhibit NF-kB activation factor alpha receptor 1-TRADD signaling Stat 1 as a component of tumor necrosis. Mol Cell Biol 20:4505-4512. CrossRef Medline

Yurco P, Cameron DA (2005) Responses of Müller glia to retinal injury in adult zebrafish. Vision Res 45:991-1002. CrossRef Medline 